\title{
Experimental Investigation of Turbulent Momentum Transfer in a Neutral Boundary Layer over a Rough Surface
}

\author{
Severine Tomas · Olivier Eiff • Valery Masson
}

Received: 12 August 2009 / Accepted: 18 November 2010 / Published online: 28 December 2010

(C) The Author(s) 2010. This article is published with open access at Springerlink.com

\begin{abstract}
The turbulent characteristics of the neutral boundary layer developing over rough surfaces are not well predicted with operational weather-forecasting models. The problem is attributed to inadequate mixing-length models, to the anisotropy of the flow and to a lack of controlled experimental data against which to validate numerical studies. Therefore, in order to address directly the modelling difficulties for the development of a neutral boundary layer over rough surfaces, and to investigate the turbulent momentum transfer of such a layer, a set of hydraulic flume experiments were carried out. In the experiments, the mean and turbulent quantities were measured by a particle image velocimetry (PIV) technique. The measured velocity variances and fluxes $\left(\overline{u_{i}^{\prime} u_{j}^{\prime}}\right)$ in longitudinal vertical planes allowed the vertical and longitudinal gradients $(\partial / \partial z$ and $\partial / \partial x)$ of the mean and turbulent quantities (fluxes, variances and third-order moments) to be evaluated and the terms of the evolution equations for $\partial e / \partial t$, $\partial \overline{u^{\prime 2}} / \partial t, \partial \overline{w^{\prime 2}} / \partial t$ and $\partial \overline{u^{\prime} w^{\prime}} / \partial t$ to be quantified, where $e$ is the turbulent kinetic energy. The results show that the pressure-correlation terms allow the turbulent energy to be transferred equitably from $\overline{u^{\prime 2}}$ to $\overline{w^{\prime 2}}$. It appears that the repartition between the constitutive terms of the budget of $e, \overline{u^{\prime 2}}, \overline{w^{\prime 2}}$ and $\overline{u^{\prime} w^{\prime}}$ is not significantly affected by the development of the rough neutral boundary layer. For the whole evolution, the transfers of energy are governed by the same terms that are also very similar to the smooth-wall case. The PIV measurements
\end{abstract}

S. Tomas · V. Masson

Centre National de Recherches Météorologiques-GAME,

Météo-France/CNRS, Toulouse, France

S. Tomas $(\bowtie)$

Cemagref, UMR G-EAU, LERMI, Aix-en-Provence, France

e-mail: severine.tomas@ cemagref.fr

O. Eiff

University of Toulouse,

INPT, UPS, IMFT (Institut de Mécanique des Fluides de Toulouse),

Toulouse, France

O. Eiff

CNRS, IMFT, Toulouse, France 
also allowed the spatial integral scales to be computed directly and to be compared with the dissipative and mixing length scales, which were also computed from the data.

Keywords Functional lengths · Integral scales · Neutral boundary layer · Particle image velocimetry measurements $\cdot$ Rough walls $\cdot$ Turbulent budgets

\section{Introduction}

Roughness effects on turbulent flow over surfaces have been studied since the mid-nineteenth century. For the atmospheric boundary layer, roughness effects have also received particular attention; see for example the review of Raupach et al. (1991). When the roughness elements are high enough, the offset constant in the smooth-wall logarithmic law changes, as discussed in most text books (e.g., Schlichting and Gersten 1979). The roughness effects are accounted for in the logarithmic law via differently defined but equivalent roughness parameters: (i) the roughness length, used mostly in the atmospheric context, (ii) the equivalent sand roughness, or finally (iii) the roughness function (see Jiménez 2004). Yet an important and long-standing question is whether the outer-layer flow $\left(z^{+}=u_{*} z / v>50\right.$, where $u_{*}$ is the friction velocity, $z$ is height and $v$ is the kinematic viscosity) (e.g. Pope 2000) depends on the wall roughness. Some studies, investigating the effect of roughness on the turbulence structure (Antonia and Luxton 1971; Bandyopadhyay 1987; Raupach et al. 1991), conclude that there is an outerlayer similarity over smooth and rough walls. In this context, Townsend (1976) similarity hypothesis is often invoked as an implicit "wall-similarity" (Raupach et al. 1991) deduced from the general Reynolds number similarity (Townsend 1976). The recent experimental studies of Flack et al. (2005) and Schultz and Flack (2007) support the outer-layer similarity of smooth- and rough-wall boundary layers in terms of both the mean flow and the Reynolds stresses. Similarly, the combined experimental and numerical investigation of Krogstad et al. (2005) suggests an outer layer that is little affected by the roughness elements. However, an outer-layer structure unaffected by the wall condition is not always found. For example, the experimental study of Krogstad et al. (1992) showed that the roughness at the wall influences the mean velocity and the turbulent stresses in the outer layer.

In a recent review, Jiménez (2004) readdressed this issue, and stressed that, in addition to the roughness Reynolds number usually considered, i.e. $R e_{*}=k_{s} u_{*} / \nu$, where $k_{s}$ is the equivalent sand roughness, care needs to be taken to account for the fact that the roughness sublayer will increasingly affect a significant portion of the logarithmic zone for low blockage ratios, $\delta / z_{h}$, where $\delta$ is the boundary-layer height and $z_{h}$ is the height of the roughness elements. Jiménez (2004) estimated that to be free from direct roughness effects, $\delta / z_{h}>40$. Thus, in order to attain fully rough regimes, the roughness Reynolds number needs to be greater than about 80 . He concludes that the Reynolds number $R e_{\delta^{+}}=\delta u_{*} / v$ (also called Kármán number $\delta_{+}$) should be greater than 4,000 . These are conditions that are usually satisfied in real atmospheric boundary-layer flows, with the possible exception of the urban boundary layer.

Because of the development of remote sensing technologies, in-situ measurements have become accurate enough to investigate turbulence in the atmospheric boundary layer (e.g. Poulos et al. 2002; Drobinski et al. 2007; Kunkel and Marusic 2006). The experiments of Kunkel and Marusic (2006) over the western Utah Great Salt Lake desert covered very high Kármán numbers $\left(\delta^{+} \cong 10^{6}\right)$ with very small relative roughness heights $\left(k_{s} / \delta \cong 10^{-4}\right)$. The results show good agreement with the similarity formulations (Marusic and Kunkel 2003) based on Townsend's attached eddy model (1976) and thus support Townsend's outer-layer 
similarity. As the authors suggest, some of the observed outer-layer differences in comparison with other studies might be attributed to blockage effects and/or low Reynolds numbers.

Yet, most boundary-layer studies, especially those concerned with roughness effects, only consider the fully developed boundary layer at equilibrium. The development of boundary layers is usually only considered in the context of a transition of a well-developed boundary layer subject to a sudden change of wall roughness, also referred to as an internal boundary layer, recent examples including those of Cheng and Castro (2002a,b) and Belcher et al. (2003). One exception is the study of Castro (2007) who examined the boundary layer developing in a wind tunnel over various roughnesses at relatively high Reynolds numbers. However, he focused only on the mean flow and concluded that outer-layer flow similarity holds even for surprisingly low blockage ratios, for $\delta / z_{h}>5$, (i.e. significantly smaller than the criterion suggested by Jiménez (2004)), but did not examine the turbulence structure nor the functional and integral length scales.

A better knowledge of the turbulence structure, including the functional and integral length scales, is necessary to validate and improve numerical simulations. This includes one-dimensional (1D) boundary-layer prediction models that are computationally inexpensive but need to be improved. For example, some 1D models still use simple expressions for the mixing length in the neutral boundary layer, such as the Blackadar mixing length (Blackadar 1962) $l=\kappa z l_{0} /\left(\kappa z+l_{0}\right)$ where $l_{0}$ is an asymptotic value of the order of a few hundred metres, which are not appropriate. This mixing length only depends on height, and not on the flow (it is equal to $\kappa z$ near the ground and to $l_{0}$ high above). The large values in the free troposphere predicted by this model are not valid, however, because the stable stratification tends to strongly limit the size of the eddies. Some mixing lengths tend to correct this with stability modulating the mixing length (Bougeault and Lacarrère 1989), as in the mesoscale meteorological model Meso-NH (Lafore et al. 1998) or the operational model AROME (Bouttier 2003, 2007; Ducrocq et al. 2005; Seity et al. 2010). Unfortunately, such a mixing length parametrization does not correctly take into account the neutral case, and does not specify the presence of a thermal inversion above the boundary layer. Indeed, at the top of the atmospheric boundary layer, there is usually a significant inversion that separates the boundary layer from the free troposphere above. This is the reason why mixing lengths are often based on a criterion of stability: in stable conditions, vortices are locally inhibited and mixing lengths are respectively small. In neutral or in unstable conditions, the mixing lengths are relatively large based on the assumption that vortices reach the boundary-layer top defined by the level of high stability. While this assumption is true in unstable conditions, it is more questionable in neutral conditions where the turbulent structure is only determined by the surface friction and not related to the temperature gradient. Consequently, in a neutral boundary layer, models tend to overestimate the mixing length. Thus, since no current formulation of the mixing length takes into account the specific features of the neutral boundary layer and its streamwise vortices, except near the surface (Redelsperger et al. 2001; Drobinski et al. 2007), there appears to be no formulation that satisfactorily predicts the complete structure of the neutral boundary layer. This is, in part, due to the lack of observations under known and controlled conditions. Hess and Garratt (2002) used in-situ observations to evaluate the first-order models of Lettau (1962) and Blackadar (1962) and Blackadar (1965) and the higher-order models of Freeman and Jacobson (2002) and Xu and Taylor (1997a,b) in the neutral case. While the first-order models generally performed well, by fitting a free parameter, the second-order models performed poorly. Hess and Garratt (2002) attribute this behaviour to deviations from the idealised conditions that the higher-order models are based upon. In summary, it can be concluded that there is a need for better experimental 
data verification under controlled conditions, with access to the turbulent structure of the boundary layer.

For this purpose, it is proposed here to experimentally investigate the development of a neutral boundary layer under a potential free-stream flow over a rough surface. The study aims to provide an idealised and simple case for analysis and validation, for which no dataset under controlled and known conditions is available. In order to attain high enough Reynolds numbers, while retaining small enough relative roughness lengths (Jiménez 2004), the flow was simulated in the large water flume of the French National Center of Meteorological Research Center in Toulouse. Vertical two-dimensional velocity fields, spanning the development of the boundary layer, were measured via a particle image velocimetry (PIV) technique. The resulting experimental data describe the inertial and outer layer, i.e. above the surface layer, of a fully rough and developing neutral boundary layer, in terms of the mean and turbulent quantities under known conditions with a high level of accuracy. The measured velocity variances and fluxes $\left(\overline{u^{\prime 2}}, \overline{w^{\prime 2}}\right.$ and $\left.\overline{u^{\prime} w^{\prime}}\right)$ in a longitudinal vertical plane allow the vertical and longitudinal gradients $(\partial / \partial z$ and $\partial / \partial x)$ of the mean and turbulent quantities (fluxes, variances and third-order moments) to be evaluated. They also enable the advection, the dynamical production, the turbulent transport, the dissipation and pressure-correlation terms of the $\partial e / \partial t, \partial \overline{u^{\prime 2}} / \partial t, \partial \overline{w^{\prime 2}} / \partial t$ and $\partial \overline{u^{\prime} w^{\prime}} / \partial t$ evolution equations to be estimated. Spatial correlation analysis permits the longitudinal and vertical integral lengths to be estimated and to be compared to dissipation lengths and mixing lengths evaluated directly from the data. The intent is to improve the parametrizations of functional lengths when equilibrium is reached. The data should also help assess LES simulations or other closure model simulations of a developing atmospheric-type rough wall neutral boundary layer.

The experimental procedure is presented in Sect. 2 and the boundary-layer development is discussed in Sect. 3. Then, the study focuses on the turbulent energy budgets and the Reynolds stresses. The spatial integral scales as well as the dissipative and mixing lengths are discussed and compared in Sect. 5, with main conclusions summarised and discussed in Sect. 6.

\section{Experimental Procedure}

\subsection{Boundary-Layer Flow Facility}

The experiments were carried out in a large, wide and high hydraulic flume ( $22 \mathrm{~m} \mathrm{long}, 3 \mathrm{~m}$ wide and $1 \mathrm{~m}$ high) (Fig. 1a) to obtain high Reynolds numbers, to generate a two-dimensional flow and to minimise other boundary effects. The flume was equipped with a series of grids to reduce the incident turbulence intensity and homogenise the incident flow, such that it can be considered uniform and potential. A 1-m long smooth surface behind the last grid was installed to allow the uniform and potential flow with a small smooth and laminar boundary layer to approach the roughness elements. A turbulent and rough boundary layer was then allowed to develop over a $12.3 \mathrm{~m}$ long and $3 \mathrm{~m}$ wide horizontal rough surface. The temperature of the water was maintained within $\pm 0.2 \mathrm{~K}$, ensuring neutral flow.

A laser Doppler velocimetry (LDV) technique was used for an initial estimation of the rough-wall boundary-layer thickness and for the validation of the flume characteristics. Vertical profiles of the mean velocity and turbulent intensity were obtained at locations $(x=$ $[-0.16 ; 0.1 ; 6 ; 10.7 ; 12.4] \mathrm{m})$ for three transverse positions $(y=[-0.5 ; 0 ; 0.5] \mathrm{m})$. For the incident free-stream velocity magnitude chosen $\left(\bar{U}_{\infty, 0}=0.35 \mathrm{~m} \mathrm{~s}^{-1}\right)$, the turbulent intensity 


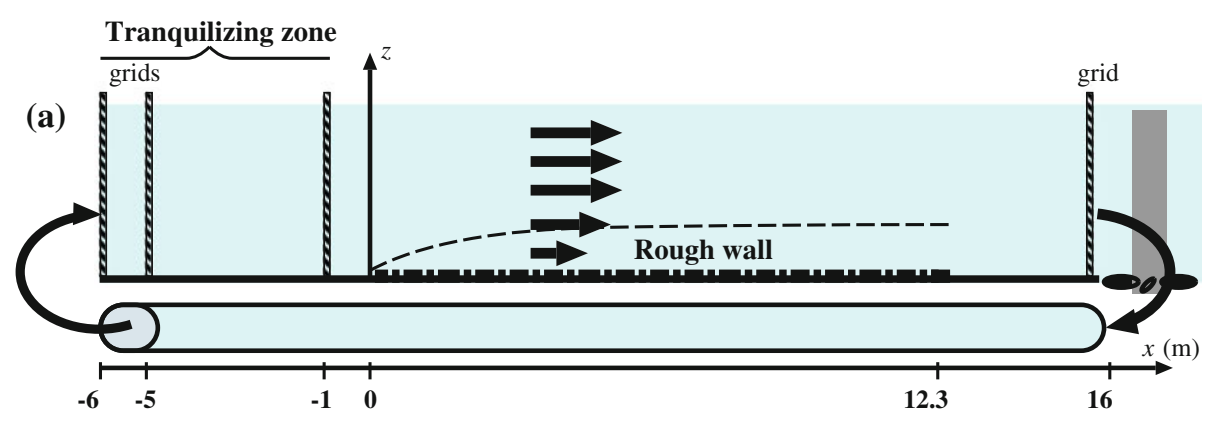

(b)

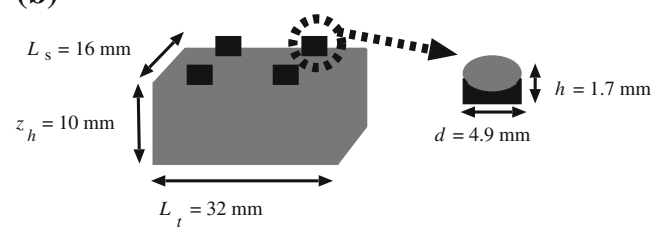

(c)

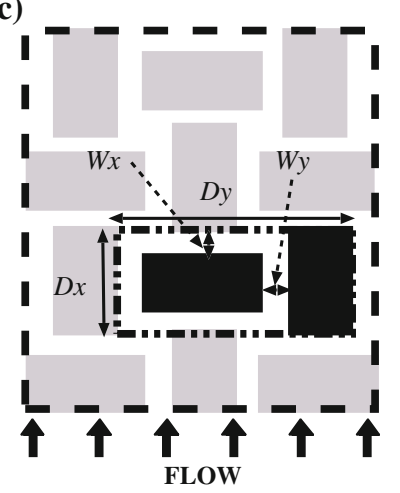

Fig. 1 (a) Sketch of the experimental set-up. (b) Typical element, LEGO ${ }^{\odot}$. (c) Arrangement of the roughness elements

and the velocity profiles for the three $y$ positions are essentially superimposed for each $x$ position. The mean deviation of these profiles is about $1 \%$ for both the mean velocity and for the intensity suggesting that the mean flow is essentially two dimensional. This is later confirmed by a very small residual of the divergence $\partial \bar{U} / \partial x+\partial \bar{W} / \partial z$ obtained from the PIV results (about $0.001 \mathrm{~s}^{-1}$ ). Also, the LDV measurements reveal that the free-stream turbulence intensity, defined by $1 / 2\left(\sqrt{\overline{u^{\prime 2}}} / \bar{U}+\sqrt{\overline{w^{\prime 2}}} / \bar{W}\right)$, is less than $1 \%$, such that freestream flow can be regarded as potential.

\subsection{Roughness Elements}

The roughness elements comprised LEGO $^{\odot}$ blocks $31.7 \mathrm{~mm}$ long, $15.7 \mathrm{~mm}$ wide and $9.6 \mathrm{~mm}$ high (Fig. 1b) to create a fully rough regime $\left(R e_{*}=u_{*} z_{h} / v \cong 200\right)$. The arrangement (Fig. 1c) was chosen to represent a medium roughness length, $z_{0}$, the roughness parameter usually used in the atmospheric context and defined by the logarithmic law:

$$
\frac{\bar{U}}{u_{*}}=\frac{1}{\kappa} \ln \left(\frac{z-z_{d}}{z_{0}}\right)
$$

where $\kappa$ is the Von Kármán's constant, fixed at 0.4 , and $z_{d}$ the zero-plane displacement. The roughness length is a hydrodynamic quantity related to the drag on the roughness elements, which is usually estimated via purely geometrical parameters in terms of the plane area density $\left(\lambda_{p}=A_{p} / A_{t}\right)$ and the frontal area density $\left(\lambda_{f}=A_{f} / A_{t}\right)$ (e.g. Raupach et al. 1980; Coceal and Belcher 2004). Here, $A_{t}$ is the area of the reference domain (here $D_{x} \times D_{y}$ ), $A_{p}$ is 
Table 1 Dimensions of the reference domain (streamwise $D_{x}$ and crosswise $D_{y}$ ), the spacing between the roughness elements contained in the domain (streamwise $W_{x}$ and crosswise $W_{y}$ ), the dimensions of the roughness elements (height $z_{h}$, length $L_{t}$ and width $L_{S}$ ), the different areas (the area of the reference domain $A_{t}$, the plane area of the obstacles viewed from above and contained in the domain of reference $A_{p}$, and the frontal area of each obstacle exposed to the wind $A_{f}$ ) and densities (the plane area density $\lambda_{p}$ and the frontal area density $\lambda_{f}$ ) (all units in $\mathrm{mm}$ )

\begin{tabular}{|c|c|c|c|c|c|c|c|c|c|c|c|}
\hline \multicolumn{2}{|c|}{ Domain } & \multicolumn{2}{|c|}{ Spacings } & \multicolumn{3}{|c|}{ Obstacles } & \multicolumn{3}{|l|}{ Areas } & \multicolumn{2}{|c|}{ Densities } \\
\hline$D_{x}$ & $D_{y}$ & $W_{x}$ & $W_{y}$ & $z_{h}$ & $L_{t}$ & $L_{S}$ & $A_{t}$ & $A_{p}$ & $A_{f}$ & $\lambda_{p}$ & $\lambda_{f}$ \\
\hline 32 & 61.3 & 8 & 8 & 10 & 32 & 16 & 1.962 & 1.024 & 480 & 0.5 & 0.2 \\
\hline
\end{tabular}

Table 2 Roughness parameters $z_{d}$ and $z_{0}$ and the ratios $z_{d} / z_{h}$ and $z_{0} / z_{h}$ obtained from the predictions based on analytical theories

\begin{tabular}{lllll}
\hline & Lettau (1969) & Kondo and Yamazawa (1986) & Bottema $(1995,1997)$ & Macdonald et al. (1998) \\
\hline$z_{0}(\mathrm{~mm})$ & 1.1 & 1.2 & 0.01 & 0.25 \\
$z_{0} / z_{h}$ & 0.11 & 0.12 & 0.001 & 0.025 \\
$z_{d}(\mathrm{~mm})$ & NA & NA & 6 & 7 \\
$z_{d} / z_{h}$ & NA & NA & 0.66 & 0.76 \\
\hline
\end{tabular}

the plane area of the obstacles viewed from above and contained in the domain of reference, and $A_{f}$ is the frontal area of each obstacle exposed to the flow (see Fig. 1b,c and Table 1 for the dimensions).

For later comparison with the measurements and for the initial design of the surface layout, several models to estimate the roughness length $z_{0}$, as well as the displacement length $z_{d}$, based on the above mentioned geometrical densities, were evaluated. The results, based on the models of Kondo and Yamazawa (1986), Bottema (1995, 1997), Lettau (1969) and Macdonald et al. (1998), are given in Table 2. The estimations of $z_{d}$, proposed by Bottema $(1995,1997)$ and Macdonald et al. (1998), where an empirical drag coefficient is also taken into account, are similar. The purely geometrical methods, developed by Kondo and Yamazawa (1986) and Lettau (1969) seem to overestimate the modelling of $z_{0}$ in comparison with the methods of Bottema $(1995,1997)$ and Macdonald et al. (1998). The latter values of $z_{0}$ are close to the desired value of $z_{0} / \delta$ for a medium roughness surface.

\subsection{PIV Measurements}

The PIV experiments were performed at a free-stream flowspeed, incident on the roughness element, $\bar{U}_{\infty, 0}$, of $0.35 \mathrm{~m} \mathrm{~s}^{-1}$. In order to characterise the upstream flow and the boundarylayer development over the rough surface, measurements were performed at six $x$ positions. The flow fields, $0.5 \mathrm{~m}$ high and $0.6 \mathrm{~m}$ wide in order to capture and exceed the maximum boundary-layer depth, were centered at $x=[0.2,1,3,6,8,11] \mathrm{m}$ with $R e_{x}=\bar{U}_{\infty} x / v=$ $\left[5 \times 10^{4}, 3 \times 10^{5}, 1 \times 10^{6}, 2 \times 10^{6}, 3 \times 10^{6}, 4 \times 10^{6}\right]$, respectively. The images were taken in the centre of the channel $(y=0)$ with a X-STREAM VISION 10 bit CMOS camera with a $1260 \times 1024$ pixel resolution and equipped with a NIKKOR camera lens with a 50- mm focal length. Image acquisition was controlled by a frequency generator that allowed the acquisition of $N$ pairs of images at $1 \mathrm{~Hz}$. A typical time interval between the two images of a pair, $\Delta t$, was about $0.017 \mathrm{~s}$. A high number of pairs was chosen to ensure convergence of 
the turbulent statistics $(N=999)$. The water was seeded with $60 \mu \mathrm{m}$ polyamide particles $\left(\right.$ Orgasol $\left.^{\circledR} 2000\right)$ illuminated with a laser light sheet $5 \mathrm{~mm}$ thick, created with an oscillating mirror and a $25 \mathrm{~W}$ Spectra Physics Beamlok argon-ion laser.

The PIV images were processed with the algorithms and interface developed by Fincham and Spedding (1997) and Fincham and Delerce (2000), which permits the effective elimination of the peak-locking bias errors. A resolution of 2 pix mm$^{-1}$ with a correlation box size of 20 pixels and a grid spacing of 10 pixels in the $x$ direction, and a correlation box size of 15 pixels and a grid spacing of 8 pixels in $z$-direction, yields a longitudinal resolution of about $5 \mathrm{~mm}$ and a vertical resolution of $4 \mathrm{~mm}$, respectively. This resolution is roughly equivalent to the laser light thickness, thus giving an essentially isotropic measurement resolution [without significant loss of out-of-plane moving particles, estimated at less than $10 \%$, well below the limit of 30\% suggested by Fincham and Spedding (1997)].

Longitudinal spatial averaging of the measured velocity fields at a given $x$ position was performed to further increase statistical convergence. It is, however, only justified for the further downstream $x$ positions $(x>3 \mathrm{~m})$. At the beginning of the rough surface $(x=0.2 \mathrm{~m})$, the velocity field is sub-divided into three parts (smooth, transition, rough) and $x$-averaged for each one. The fluctuations $\left(u_{i}^{\prime}\right)$ in each case are the difference between the raw field $\left(u_{i}\right)$ and the spatially- and temporally-averaged field $\left(\overline{U_{i}}\right)$, which is given by:

$$
\overline{U_{i}}(z) \equiv \frac{1}{N} \sum_{p=1}^{N}\left[\frac{1}{n_{x}} \sum_{q=1}^{n_{x}} u_{i}(q, z, p)\right]
$$

where $N$ is the number of computed velocity fields $(N=999)$ and $n_{x}$ is the number of velocity vector columns used ( $n_{x}=90$, for a complete field).

The integral time scale can be estimated to be larger than the advection time (i.e., Taylor's hypothesis with $u^{\prime} / U \ll 1$ where advection dominates). Therefore, a conservative estimate based on the maximum advection time and the sampling frequency of $1 \mathrm{~Hz}$ yields a roughly $50 \%$ overlap of the large scales between consecutive instantaneous velocity fields, or about 2,000 independent samples at large scales. This is larger than the 1,000 large scales suggested by Tennekes and Lumley (1972), for example, to ensure statistical convergence. This sampling is higher than the direct numerical simulations (DNS) of Coceal et al. (2006) who averaged over only 400 large scales. It should also be noted that the increased spatial dispersion, as the top of the roughness elements is approached (i.e. the roughness sublayer), prevents representative mean statistics being evaluated with single vertical plane measurements. If meaningful statistics in the roughness sublayer were desired, several vertical planes across a typical roughness area would need to be measured to allow spatial (double-) averaging in all horizontal planes $(x, y)$. This was not the objective of our study, so the measurements and statistical evaluations were limited to above the roughness layer (taken as $z>2 z_{h}$, e.g. Macdonald 2000).

\section{Boundary-Layer Development}

\subsection{Mean Velocity Field}

In this section, the mean velocity fields as the boundary layer develops over the rough surface are presented. The logarithmic law scaling parameters used to normalise the velocity and length quantities are also determined. 

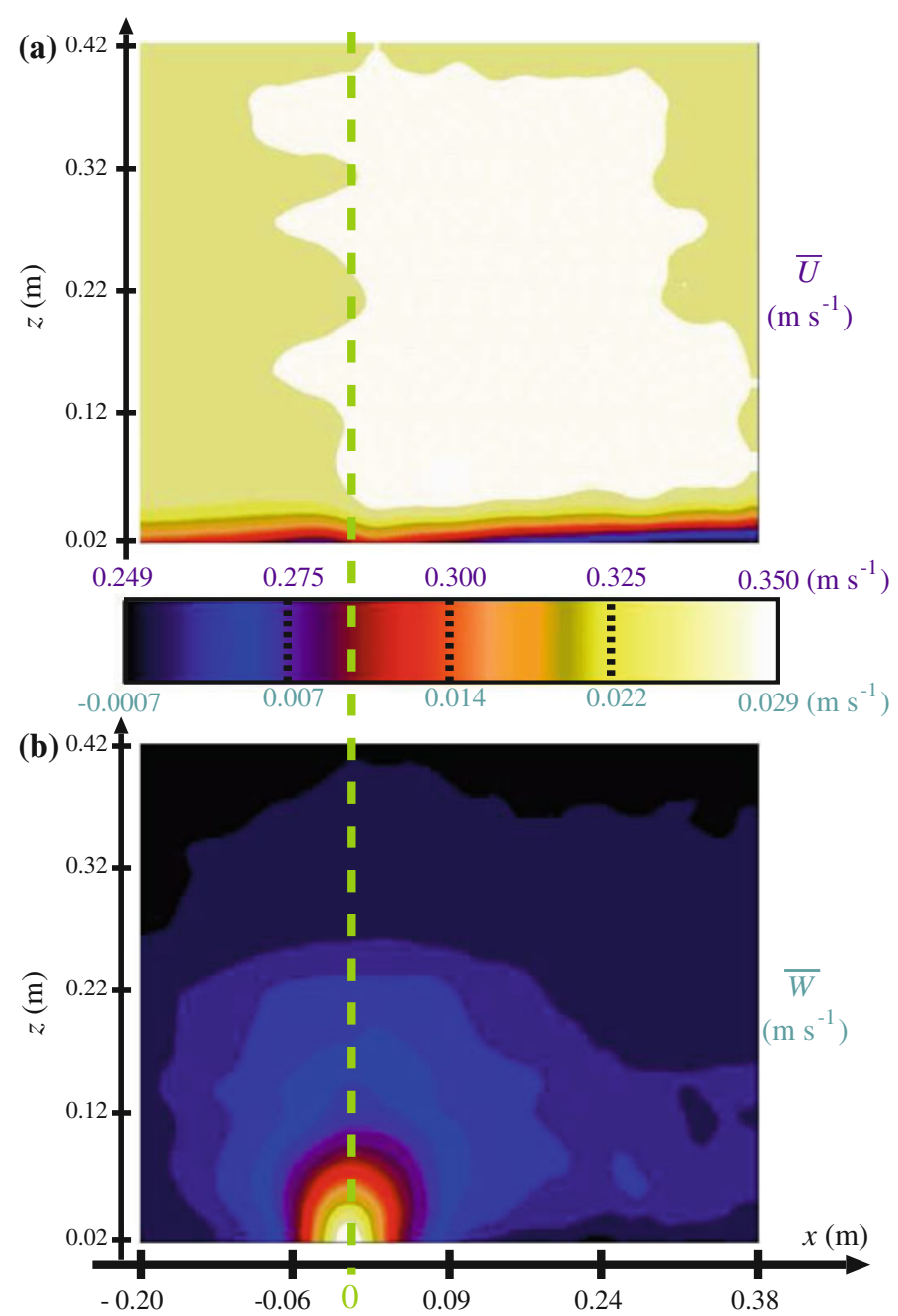

Fig. 2 Mean velocity fields at position A $(x=0)$, near the commencement of the roughness bed (a) $\bar{U}(x, z)$ and $(\mathbf{b}) \bar{W}(x, z)$

Figure $2 \mathrm{a}$ and $\mathrm{b}$ shows the temporally-averaged longitudinal and vertical velocity fields, $\bar{U}(x, z)$ and $\bar{W}(x, z)$, respectively, around the beginning of the rough bed $(x=0)$. In the longitudinal velocity field $\bar{U}(x, z)$ for $x>0$, the development of the rough boundary layer can be seen, as can the incident smooth boundary layer, which is relatively thin, as desired, and of depth of the order of the roughness elements $\left(\delta_{\text {smooth }} / z_{h} \cong 1\right)$. The ensuing flow can thus be considered as a new boundary layer that starts to develop over the roughness elements under a potential and uniform free stream, as opposed to beneath an existing boundary layer. The effect of the surface roughness discontinuity, at $x=0$, can clearly be seen in the strong $\bar{W}$ perturbation centred around $x=0$, with a strong positive increase in $\bar{W}$ from effectively zero to about $0.03 \mathrm{~m} \mathrm{~s}^{-1}$. This rise is symmetric with respect to the discontinuity at $x=0 \mathrm{~m}$, with a radius of influence of about $0.18 \mathrm{~m}$ or about $10 z_{h}$. Also, since around the 


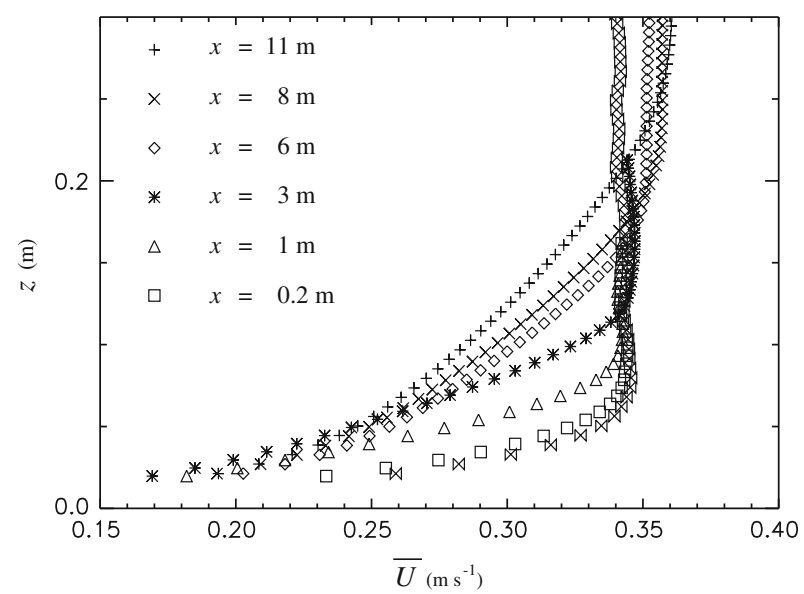

Fig. 3 Mean longitudinal velocity profiles $\bar{U}(z)$ at all measurement $x$ positions (A-F)

discontinuity the flow over the obstacles must first accelerate when deviated, an initial acceleration of $\bar{U}(\partial \bar{U} / \partial x>0)$ occurs with an accompanying boundary-layer height decrease, as is indeed observed in Fig. 2a. Further downstream $(x>0.03 \mathrm{~m})$, the new boundary-layer growth dominates due to the drag exerted, and $\partial \bar{U} / \partial x<0$ while $\partial \delta / \partial x>0$.

Figure 3 shows the mean longitudinal velocity profiles obtained via time and spatial averaging at each measurement station $(\mathrm{A}-\mathrm{F})$. Because of the finite depth of the water in the flume, the boundary-layer growth is associated with a slight increase in the free-stream velocity $\left(\bar{U}_{\infty}\right)$ to balance the flow rate due to the increasing displacement thickness in the boundary layer. This increase in $\bar{U}_{\infty}$ is small, about $5 \%$ over all measurement stations, and in agreement with the preliminary LDV measurements (Sect. 2.1).

The mean streamwise velocity profile in the log-law region given by Eq. 1 can be used to determine the roughness length $z_{0}$ and the friction velocity $u_{*}$. To evaluate the displacement height $z_{d}$ independently, we chose Macdonald (2000) semi-empirical relation,

$$
z_{d}=\left[1+\alpha^{-\lambda_{p}}\left(\lambda_{p}-1\right)\right] z_{h}
$$

where $\alpha$ is an empirical coefficient, $C_{d}$ is the drag coefficient fixed at 1.2 with a corrective factor $\beta$. For cubical and staggered obstacles, Macdonald et al. (1998) give $\alpha=4.43$ and $\beta=1$, which yields $z_{d}=0.76 z_{h}$. Macdonald (2000) has verified the results of Raupach et al. (1980) and concluded that the log-law region, characterised by Eq. 1, is valid from $z=2.3 z_{h}$ to $z=3.5 z_{h}$. Thus, by linear regression in the region $2.3 z_{h}<z<3.5 z_{h}$, for $x=[6,8$, 11] $\mathrm{m}, u_{*}$ and $z_{0}$ were obtained (Table 3 ). The roughness length $z_{0}$ and the friction velocity $u_{*}$ for all values of $x>6 \mathrm{~m}$ were found to be approximately constant: $0.25 \pm 0.05 \mathrm{~mm}$ and $0.02 \pm 0.001 \mathrm{~m} \mathrm{~s}^{-1}$, respectively. The value of $z_{0}$ agrees with the semi-empirical prediction established by Macdonald et al. (1998) reported earlier in Table 2. For all values of $x<6 \mathrm{~m}$ (positions $\mathrm{A}$ to $\mathrm{C}$ ), estimating $u_{*}$ and $z_{0}$ with the log law is not expected to be valid since the log-law region is too small for a relatively large $z_{h} / \delta$, as discussed earlier.

Another way of determining $u_{*}$, independent of the log law, is by assuming $u_{*}{ }^{2}=$ $-{\overline{u^{\prime} w^{\prime}}}_{z=z_{d}}$. To evaluate the Reynolds stress in the canopy at $z=z_{d}$, which was not measured at this height, we can either extrapolate the linear stress variation in the outer layer or the essentially constant-stress part below $(z / \delta<0.2)$, as shown in a typical Reynolds stress profile in Fig. 4a. Here, we chose to take an average of the obtained minimum and maximum 
Table 3 Estimation of the parameters $z_{0}$ and $u_{*}$, based on linear regression of the logarithmic law in the region $2.3 z_{h}<z<3.5 z_{h}$ and for $x>6 \mathrm{~m}$ with $z_{d}=7.6 \mathrm{~mm}$, for $\bar{U}_{\infty, 0}=0.35 \mathrm{~m} \mathrm{~s}^{-1}$

\begin{tabular}{llll}
\hline & $x(\mathrm{~m})$ & & \\
\cline { 2 - 4 } & 6 & 8 & 11 \\
\hline$u_{*}\left(\mathrm{~m} \mathrm{~s}^{-1}\right)$ & 0.020 & 0.021 & 0.019 \\
$z_{0}(\mathrm{~mm})$ & 0.253 & 0.346 & 0.223 \\
\hline
\end{tabular}

(a)

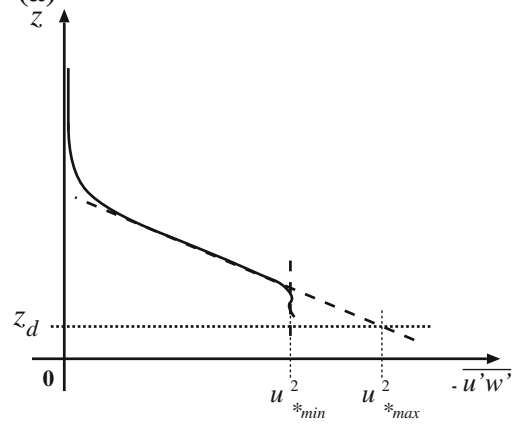

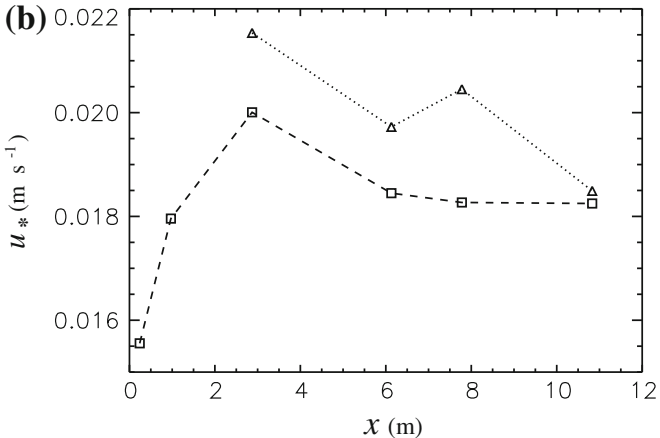

Fig. 4 (a) Method used to estimate the friction velocity $u_{*}^{2}=-\overline{u^{\prime} w^{\prime}} z=z_{d}$ from the $\overline{u^{\prime} w^{\prime}}$ profiles: the minimum value is obtained by averaging the first points of the profile (for $z / \delta<0.2$ ) and the maximum value is given by the intersection between the extrapolation of the linear regression of $\overline{u^{\prime} w^{\prime}}$ for $0.5<z / \delta<0.9$ and $z=z_{d}$. (b) Squares symbols friction velocity $u_{*}$ estimated by method (a) with $u_{*}=\left(u_{* \max }+u_{* \max }\right) / 2$ via $-\overline{u^{\prime} w^{\prime}}$ shear-stress profiles, and triangle symbols friction velocity $u_{*}$ estimated via the logarithmic law fits in the region $2.3 z_{h}<z<3.5 z_{h}$ (see Table 3)

$u_{*}$ values, shown in Fig. $4 \mathrm{~b}$ (squares) with those obtained from the log law (triangles). It can be seen that the friction velocity decreases with fetch until an equilibrium value is reached, as already observed by Rao et al. (1974) for a boundary layer over a change of surface roughness. In the case of a newly developing boundary layer under a free-stream flow, a detailed discussion of the evolution of $u_{*}$ is given in Castro (2007). It can be noted that the flow is fully rough as desired, since $z^{+}=\frac{z_{0} u_{*}}{v} \geq 2$, or equivalently $k_{s}^{+}=\frac{k_{s} u_{*}}{v} \geq 70$ with $k_{s}=z_{0} / 0.033$ (e.g. Jiménez 2004).

\subsection{Vertical Development of the Boundary Layer}

The boundary-layer height, $\delta(x)$, has been determined via two methods: firstly, as the height where $\bar{U}$ reaches $99 \%$ of the free-stream velocity magnitude $\left(\bar{U}_{\infty}\right)$, and secondly as the height where the flux $-\overline{u^{\prime} w^{\prime}}$ decreases to $5 \%$ of its maximum value. The boundary-layer height based on these two definitions and normalised by $z_{0}$ is shown in Fig. 5; values can be seen to be in close agreement although the height based on the mean velocity is consistently higher than the height based on the turbulent flux, a sign of both the robustness and the arbitrary nature of both criteria. This led to the use of the average of both criteria to fit the evolution of the boundary-layer height, yielding:

$$
\frac{\delta}{z_{0}}\left(\frac{x}{z_{0}}\right)=0.18\left(\frac{x}{z_{0}}\right)^{0.79}-203 .
$$




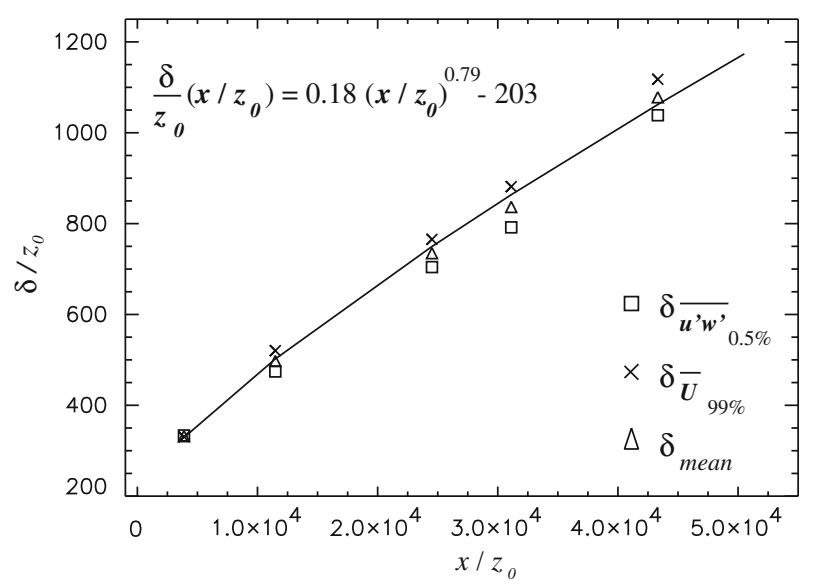

Fig. 5 Boundary-layer height evolution $(\delta)$ normalized by the roughness length $\left(z_{0}\right)$, the square symbols $5 \%$ of $\overline{u^{\prime} w_{\max }^{\prime}}$ criterion; the $X$ symbols $99 \%$ for the $\overline{U_{\infty}}$ criterion; the triangle symbols average of $\delta \overline{u^{\prime} w^{\prime} 5 \%}$ and $\delta_{\bar{U}}$; $;$ the solid line the best fit of the average of both criteria

As expected, this law differs from the classic smooth-surface turbulent boundary-layer growth rate that predicts a power-law exponent of 0.85 and a coefficient of about 0.08 (White 1991). Our power-law exponent is close to the well-known 4/5th power law, however, starting with Elliot's model (1958), to predict the growth of an internal boundary layer developing after a sudden change of roughness. Numerous experimental studies of the developing internal boundary layer (e.g. Antonia and Luxton 1971; Pendergrass and Arya 1984) as well as numerical studies (e.g. Lin et al. 1997) have yielded exponents close to 0.8. Townsend's (1966) theoretical analysis shows that, for a large change in friction velocity, from a very smooth to a very rough surface, the flow behaves essentially as that of a boundary layer developing below a uniform free-stream velocity, i.e. the situation studied here. However, the coefficient and depth of the boundary layer measured is less than those predicted by Elliot's theory (1958), which is valid for small changes in friction velocity. Indeed, our measured coefficient of 0.18 is less than the value of 0.68 predicted by Elliot with an estimated smooth-surface roughness length of $z_{0_{1}}$ of $3 \times 10^{-5} \mathrm{~m}$ (Perrier and Butet 1988). In addition, our coefficient is still smaller than the 0.35 predicted by Pendergrass and Arya (1984) for a roughness change parameter $M=\ln \left(\frac{z_{0}}{z_{0}}\right)=-2.3$ which is close to our value $M=-2.1$.

\subsection{Turbulent Quantities}

We now turn our attention to the evolution of the turbulent quantities. Figure $6 a-c$ shows the profiles of $\overline{u^{\prime 2}} / u_{*}^{2}, \overline{w^{\prime 2}} / u_{*}^{2}$ and $-\overline{u^{\prime} w^{\prime}} / u_{*}^{2}$, respectively, as a function of $z / \delta$ where $\delta$ is the local mean value of the boundary-layer height (Fig. 5) and $u_{*}$ is the local value obtained from the Reynolds stress profile, for all measured $x$ positions. The normalised values of these variances and covariances, at last initially, increase with $x$. According to the study of Antonia and Luxton (1971), again for internal boundary-layer growth, the increase of $\overline{u^{\prime 2}} / u_{*}^{2}$ is linked to the intensification of the vertical gradient of $\bar{U}$, which is accompanied by an increase of dynamical production. Here, the growth in the stresses with $x$ tends to decrease until an equilibrium is reached where the profiles overlap. This equilibrium is reached between $x=3 \mathrm{~m}$ and $x=6 \mathrm{~m}\left(R e_{x}=1 \times 10^{6}\right.$ and $\left.2 \times 10^{6}\right)$ for all three stresses. 
Fig. 6 Locally normalised profiles of (a) $\overline{u^{\prime 2}} / u_{*}^{2}$, (b) $\overline{w^{\prime 2}} / u_{*}^{2}$ and (c) $\overline{u^{\prime} w^{\prime}} / u_{*}^{2}$ as a function of $z / \delta$ for all measured $x$ positions
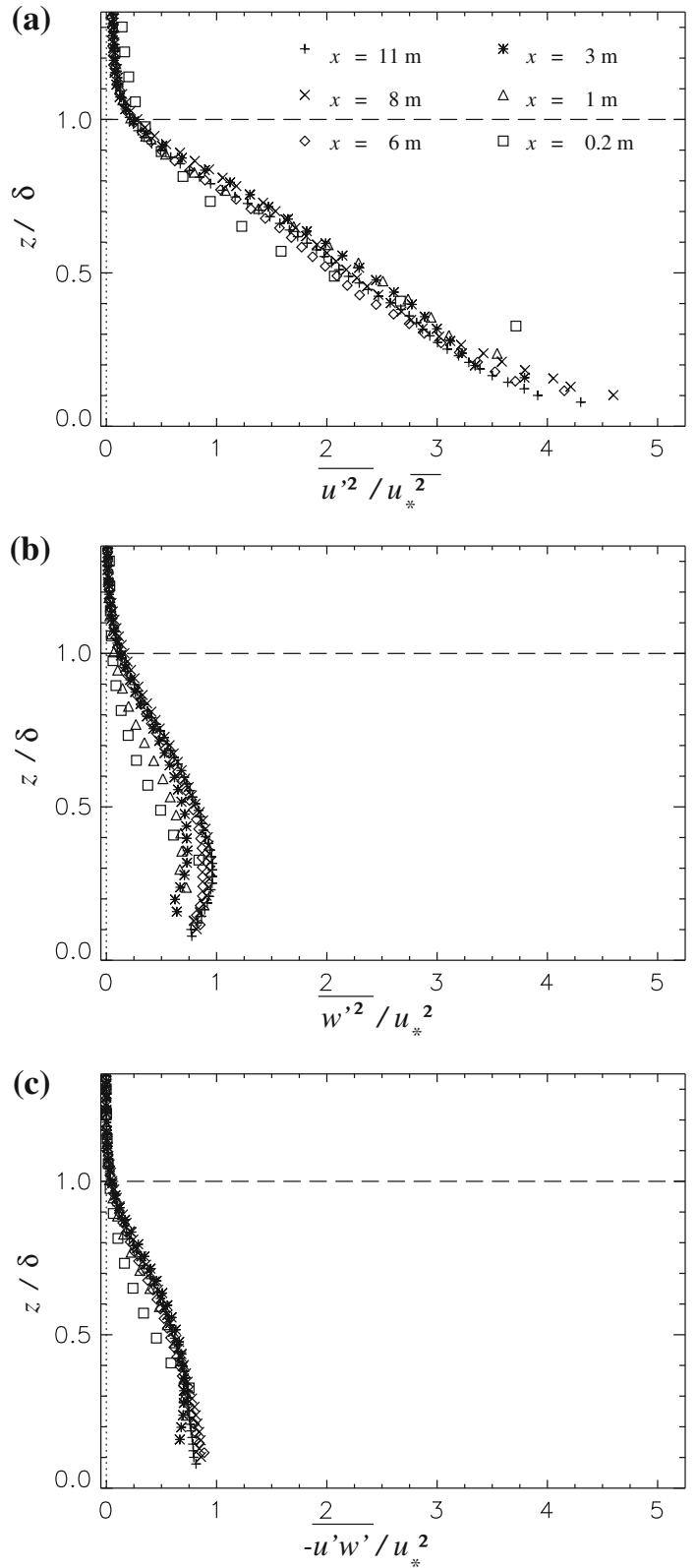

Both $\overline{u^{\prime 2}} / u_{*}^{2}$ and $-\overline{u^{\prime} w^{\prime}} / u_{*}^{2}$ are maximum near the top of roughness elements. This feature can be related to the existence of turbulent structures that are generated by the roughness elements (Kim et al. 1987; Castro et al. 2006). The existence of these structures is supported by the experimental work of Hommema and Adrian (2003). From these maxima, $\overline{u^{\prime 2}} / u_{*}^{2}$ decreases linearly up to the top of the outer layer, while the decrease of $-\overline{u^{\prime} w^{\prime}} / u_{*}^{2}$ is quasi-linear, as expected for a pressure driven flow. Yet near the rough wall, the shear stress remains roughly constant as is usually observed for rough surface, neutral boundary-layer 
measurements (Cheng and Castro 2002a,b; Chow et al. 2005; Drobinski et al. 2007). This could be attributed, as the roughness elements are approached, to a lack of sufficient spatial resolution in this high intensity region and also to possible remaining spatial dispersion not accounted for in single plane measurements as the roughness layer is approached.

Figure $6 \mathrm{~b}$ shows that the $\overline{w^{\prime 2}} / u_{*}^{2}$ profiles in equilibrium (for $x>6 \mathrm{~m}$ ) have a maximum near $z / \delta \cong 0.3$ but vary very weakly between $0<z / \delta<0.5$ around a value of about 1 . The quasi-constancy of $\overline{w^{\prime 2}} / u_{*}^{2}$ until $0.5 \delta$ agrees with the profiles observed by Panofsky (1974), Yaglom (1991) and Castro et al. (2006) and simulated by Drobinski et al. (2007) and Coceal et al. (2006). Near the surface, $(z / \delta \cong 0.1)$, the normalised variances lie between 4 and 4.5 for $\overline{u^{\prime 2}} / u_{*}{ }^{2}$ and around 0.8 for $\overline{w^{\prime 2}} / u_{*}{ }^{2}$. These values are slightly lower than those deduced from the in-situ measurements and the LES results of Drobinski et al. (2007), which lie between 5 and 6 for $\overline{u^{\prime 2}} / u_{*}^{2}$ and $1-2$ for $\overline{w^{\prime 2}} / u_{*}{ }^{2}$. Figure $6 \mathrm{a}$ and $\mathrm{b}$ reveals that $\overline{w^{\prime 2}} / u_{*}^{2}$ is about four times smaller than $\overline{u^{\prime 2}} / u_{*}^{2}$, i.e. the flow is strongly anisotropic, at large scales. It is also the ratio established by Moeng and Sullivan (1994), and by the observations of Nicholls and Readings (1979) and Grant (1986), who found $\overline{u^{\prime 2}} / \overline{w^{\prime 2}} \cong 4$.

It can be concluded that our experiment captures the turbulent structure of a developing rough surface neutral boundary layer, which reaches equilibrium between $R e_{x} \cong 1 \times 10^{6}$ and $2 \times 10^{6}(3<x<6 \mathrm{~m})$. In order to investigate the transfer processes governing such a developing boundary layer, the budgets of the turbulent kinetic energy $e, \overline{u^{\prime 2}}, \overline{w^{\prime 2}}$ and $\overline{u^{\prime} w^{\prime}}$ are discussed below.

\section{Normalised Budgets of the Second-Order Turbulent Quantities}

We examine the budgets of the second-order turbulent transport equations to help evaluate the numerical models and characterise the transfers between the turbulent quantities. The Reynolds-stress transport equation is given below, where Coriolis and thermal production effects are not considered,

$$
\begin{aligned}
\frac{\partial}{\partial t}\left(\overline{u_{i}^{\prime} u_{j}^{\prime}}\right)= & \underbrace{-\overline{U_{k}} \frac{\partial \overline{u_{i}^{\prime} u_{j}^{\prime}}}{\partial x_{k}}}_{A D V}-\underbrace{\left(\overline{u_{k}^{\prime} u_{i}^{\prime}} \frac{\partial \overline{U_{j}}}{\partial x_{k}}+\overline{u_{k}^{\prime} u_{j}^{\prime}} \frac{\partial \overline{U_{i}}}{\partial x_{k}}\right)}_{D P}-\underbrace{\frac{1}{\rho_{0}}\left(\overline{u_{i}^{\prime} \frac{\partial p^{\prime}}{\partial x_{j}}}+\overline{u_{j}^{\prime} \frac{\partial p^{\prime}}{\partial x_{i}}}\right)}_{P C} \\
& \underbrace{-2 v \overline{\left(\frac{\partial u_{i}^{\prime}}{\partial x_{k}} \frac{\partial u_{j}^{\prime}}{\partial x_{k}}\right)}}_{D I S S} \underbrace{-\frac{\partial \overline{u_{k}^{\prime} u_{i}^{\prime} u_{j}^{\prime}}}{\partial x_{k}}}_{T R} \underbrace{+v \frac{\partial^{2} \overline{u_{i}^{\prime} u_{j}^{\prime}}}{\partial x_{k} \partial x_{k}}}_{D I F F} .
\end{aligned}
$$

The index $k$ ranges from 1 to 3 and refers, respectively, to the longitudinal, transverse and vertical components. The terms on the right-hand side represent: advection $(A D V)$, dynamical production $(D P)$, pressure-correlation $(P C)$, dissipation $(D I S S)$, turbulent transport $(T R)$ and molecular diffusion $(D I F F)$. It may be noted that, what is often referred to as the dissipation in Eq. 5, is in fact the pseudo-dissipation, $\tilde{\varepsilon}$, defined by:

$$
\tilde{\varepsilon}=v \overline{\frac{\partial u_{i}^{\prime}}{\partial x_{j}} \frac{\partial u_{i}^{\prime}}{\partial x_{j}}},
$$


which can be related to the true dissipation, $\varepsilon$ by

$$
\varepsilon=\tilde{\varepsilon}+v \frac{\partial^{2} \overline{u_{i}^{\prime} u_{j}^{\prime}}}{\partial x_{i} \partial x_{j}}
$$

The diffusion term can be neglected since it is the product of the viscosity $\left(10^{-6} \mathrm{~m}^{2} \mathrm{~s}^{-2}\right)$ and second-order derivatives. As discussed in Sect. 2.1, under the present two-dimensional conditions, the transverse gradients $(\partial / \partial y)$ of the time-averaged quantities and the transverse mean velocity $(\bar{V})$ can also be neglected. Also, for $x$ stations at $1 \mathrm{~m}$ and further, the vertical mean velocity $(\bar{W})$ is negligible as discussed in Sect. 3.1. Finally, since the flow is statistically stationary, $\partial \overline{u_{i}^{\prime} u_{j}^{\prime}} / \partial t=0$.

\subsection{Turbulent Kinetic Energy Evolution Equation}

In addition to the above general approximations, in the budget of the turbulent kinetic energy $\left(e=u_{i}^{\prime} u_{i}^{\prime} / 2\right)$, the pressure-correlation term $(P C)$ can be neglected as it is expected to be a relatively weak transport term for the smooth boundary layer (Pope 2000). This implies:

$$
P C \overline{u^{\prime 2}}+P C \overline{v^{\prime 2}}+P C \overline{w^{\prime 2}} \cong 0 .
$$

Eq. 5 applied to $e$, thus reduces to

$$
0=\underbrace{-\bar{U} \frac{\partial e}{\partial x}}_{A D V} \underbrace{-\overline{u^{\prime 2}} \frac{\partial \bar{U}}{\partial x}-\overline{u^{\prime} w^{\prime}} \frac{\partial \bar{U}}{\partial z}}_{D P} \underbrace{-\tilde{\varepsilon}}_{D I S S} \underbrace{-\frac{\partial \overline{u^{\prime} e}}{\partial x}-\frac{\partial \overline{w^{\prime} e}}{\partial z}}_{T R} .
$$

Here, $\tilde{\varepsilon}$ cannot be estimated directly from the measurements, at least with reasonably accuracy, firstly, because the transverse gradients have not been measured and secondly, because the spatial resolution of $5 \mathrm{~mm}$ is too large. It is estimated to be about 16 dissipation scales $(\eta)$ via the turbulence intensities in Fig. 6 and the later integral-scale computations (Sect. 5.1). This is not sufficiently small to include the peak of the dissipation spectrum at about $24 \eta$ (Pope 2000). However, since $\tilde{\varepsilon}$ is the only unknown quantity in the measured budget (Eq. 9) it can be estimated via the residual.

Also, for evaluating the turbulent kinetic energy, $e$, an assumption needs to be made for $\overline{v^{\prime 2}}$. The measurements of Cheng and Castro (2002b) and Macdonald et al. (2002), as well as the simulations of Castro et al. (2006), show that $\overline{v^{\prime 2}} \cong 0.5\left(\overline{u^{\prime 2}}+\overline{w^{\prime 2}}\right.$ ), while the in-situ experiments of Drobinski et al. $(2004,2007)$ and the simulations of Moeng and Sullivan (1994) suggest that the coefficient is closer to 0.4. Here, the coefficient of 0.5 is retained since the studies that support this approximation have a configuration close to our experiment, notably for the characteristics of the canopy $\left(z_{0} / z_{h}\right.$ and $\left.u_{*} / U_{\infty}\right)$. It can also be noted that this uncertainty in $\overline{v^{\prime 2}}$ affects the estimation of the turbulent transport and the advection terms by a factor of $7 \%$, and the pseudo-dissipation term by about $4 \%$.

The turbulent kinetic energy budget is shown in Fig. 7a. Above $z / \delta \geq 0.1$, advection is a minor term, even smaller than the minor vertical turbulent transport term. However, the vertical turbulent transport is not negligible at the top of the boundary layer, for $0.6<z / \delta<1$. Indeed, above $z / \delta>0.6$, although weak compared to the dissipation and the dynamical production in the lower layer, it becomes the only significant source here. Advection also grows in importance, becoming a minor sink term peaking near $z / \delta \cong 0.8$. This balance between the turbulent transport and the advection agrees with the simulations of Mason and Thomson (1987) of the neutral boundary layer. In the outer layer, but below $z / \delta \cong 0.6$, the dynamical 

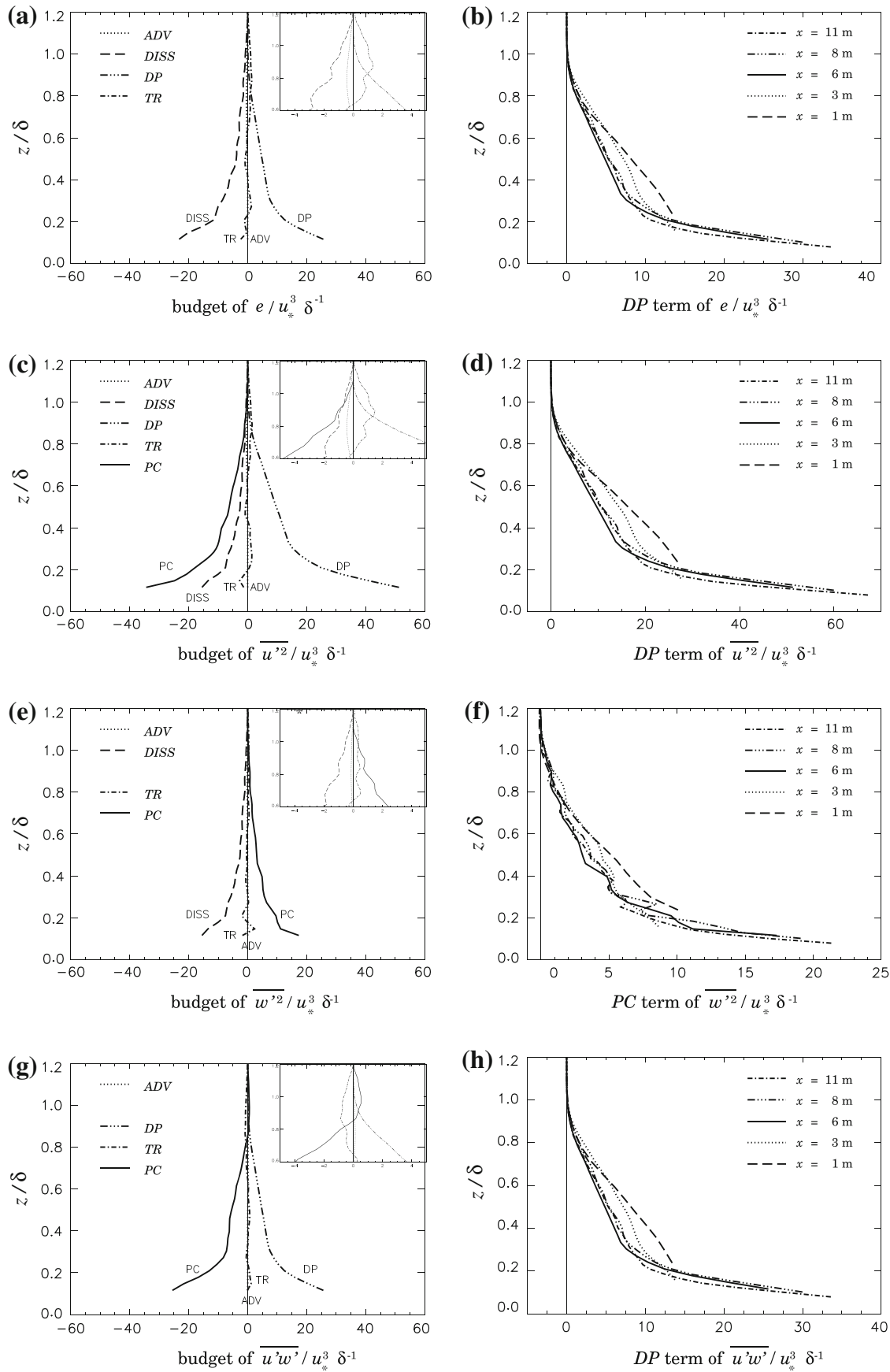

Fig. 7 Locally normalised budget of $e(\mathbf{a}), \overline{u^{\prime 2}}(\mathbf{c}), \overline{w^{\prime 2}}(\mathbf{e})$ and $\overline{u^{\prime} w^{\prime}}(\mathbf{g})$ as a function of $z / \delta$ for the D position $(x=6 \mathrm{~m})$. Dominant source terms of each budget: the dynamical production term $(D P)$ for the budget of $e$ (b), $\overline{u^{\prime 2}}(\mathbf{d}), \overline{u^{\prime} w^{\prime 2}}(\mathbf{h})$ (which equals the $D P$ for the budget of $e$ ) and the pressure-correlation term $(P C)$ for the budget of $\overline{w^{\prime 2}}$ as a function of $z / \delta$ for all $x$ positions 
production essentially balances the dissipation as observed by Glendening and Lin (2002) in large-eddy simulation of an internal boundary layer. The preponderant contribution of the dynamical production has also been assessed by Drobinski et al. (2004) and more recently by Castro et al. (2006) and Burattini et al. (2008), and is the classic assumption that stipulates that the production rate equals the dissipation. This also supports the outer-layer similarity hypothesis, the budgets being very similar as in the case of the smooth boundary layer (Pope 2000). Figure $7 \mathrm{~b}$ shows the longitudinal evolution of the dynamical production term of $e$, the most significant term of the budget of $e$ measured directly. It can be seen that for $x=1 \mathrm{~m}$ and $x=3 \mathrm{~m}$ (positions $\mathrm{B}$ and $\mathrm{C}$ ) the dynamical production is higher than for the further $x$ positions in the range $0.3<z / \delta<0.7$. This source "surplus" is balanced by an increase in the sink of the vertical turbulent transport of $e$ (not shown), which decays by $x=6 \mathrm{~m}$. By $x=6 \mathrm{~m}$ at the latest, all the profiles have converged (including the other terms, not shown) in accordance with the observation with regard to the turbulent stresses (Fig. 6a-c) that equilibrium is reached in the region $3 \mathrm{~m}<x<6 \mathrm{~m}$.

\section{$4.2 \overline{u^{\prime 2}}$ Evolution Equation}

For stationary and two-dimensional flows, Eq. 5 applied to $\overline{u^{\prime 2}}$ reduces to

$$
\begin{aligned}
0= & \underbrace{-\bar{U} \frac{\partial \overline{u^{\prime 2}}}{\partial x}}_{A D V} \underbrace{-2\left(\overline{u^{\prime 2}} \frac{\partial \bar{U}}{\partial x}+\overline{u^{\prime} w^{\prime}} \frac{\partial \bar{U}}{\partial z}\right)}_{D P} \underbrace{-\frac{2}{\rho_{0}} \overline{u^{\prime} \frac{\partial p^{\prime}}{\partial x}}}_{P C} \\
& \underbrace{-2 v\left(\left(\frac{\partial u^{\prime}}{\partial x}\right)^{2}+\left(\frac{\partial u^{\prime}}{\partial z}\right)^{2}\right)}_{\varepsilon_{u}^{\prime 2}} \underbrace{-\frac{\partial \overline{u^{\prime 3}}}{\partial x}-\frac{\partial \overline{w^{\prime} u^{\prime 2}}}{\partial z}}_{T R} .
\end{aligned}
$$

The dissipation term $\left(\varepsilon_{u}^{2}\right)$ and the pressure-correlation $(P C)$ term are the two unknown terms in the budget. Thus, in order to balance the budget, an additional hypothesis needs to be made. Pope (2000), based on DNS data of Spalart (1988) of a boundary layer over a flat smooth plate, shows that close to the wall, the anisotropy in the dissipation rate $\left(\varepsilon_{u_{i}^{\prime} u_{j}^{\prime}}\right)$ of the Reynolds stresses, $\overline{u_{i}^{\prime} u_{j}^{\prime}}$, is clearly large. However, for $z / \delta>0.1$, there is approximate isotropy. The small level of anisotropy in $\varepsilon_{u_{i}^{\prime} u_{j}^{\prime}}$ for $z / \delta>0.1$ can be attributed to the relatively low Reynolds number of the DNS of Spalart (1988). For a rough-wall boundary layer, the experiments of Saddoughi and Veeravalli (1994) and Saddoughi (1997) confirm that at this altitude there is also local isotropy of $\varepsilon_{u_{i}^{\prime} u_{j}^{\prime}}$ at high Reynolds numbers. Some studies even conclude that the roughness increases the degree of isotropy closer to the wall and this impact is greater with three-dimensional roughness (Castro et al. 2006). Therefore, as the present experiment was performed at high Reynolds number $\left(R e_{\delta^{+}}=\delta u_{*} / v>3 \times 10^{3}\right.$ at $\left.x=6 \mathrm{~m}\right)$ and with three-dimensional roughness, the dissipation rate $\left(\varepsilon_{u_{i}^{\prime} u_{j}^{\prime}}\right)$ of $\overline{u_{i}^{\prime} u_{j}^{\prime}}$ can reasonably be expressed as

$$
\varepsilon_{u_{i}^{\prime} u_{j}^{\prime}}=\frac{2}{3} \tilde{\varepsilon} \delta_{i j},
$$

where $\delta_{i j}$ is the Kronecker function. Thus, taking $\varepsilon_{u}^{\prime 2}=2 / 3 \tilde{\varepsilon}$, with $\tilde{\varepsilon}$ obtained from Eq. 9, the pressure-correlation is the only residual term of the budget of $\bar{u}^{\prime} 2$. The estimation of $\varepsilon_{u}^{\prime 2}$ 
and consequently of the pressure-correlation term is thus again essentially affected by the uncertainty in $\overline{v^{\prime 2}}$ by no more than $4 \%$.

The budget of $\overline{u^{\prime 2}}$ (Fig. 7c) shows, as expected, that the intensity of all the terms decreases as $z / \delta$ increases. The turbulence acts principally in the lower part of the outer layer, and above the surface layer, the dynamical production is the only significant source, as shown by Pope (2000) for a smooth-surface boundary layer. The advection by the mean wind or turbulent transport are not really important for the evolution of $\overline{u^{\prime 2}}$, except at the top of the outer layer where the advection peaks near $z / \delta \cong 0.8$ and where the turbulent transport becomes the dominant source, as for the budget of $e$. The pressure-correlation term is significant throughout, and is the main sink for $\overline{u^{\prime 2}}$ above the surface layer. Similarly to the boundary layer over a smooth surface (Spalart 1988), the pressure fluctuations appear to distribute the energy between the different components: from $\overline{u^{\prime 2}}$ to $\overline{v^{\prime 2}}$ and $\overline{w^{\prime 2}}$ (see below). Figure $7 \mathrm{~d}$ shows that the equilibrium of the most significant term in the $\overline{u^{\prime 2}}$ budget, the dynamical production term, is reached between $x=3 \mathrm{~m}$ and $x=6 \mathrm{~m}$, as for the budget of $e$ and in agreement with the longitudinal evolution of $\overline{u^{\prime}} / u_{*}^{2}$ (Sect. 3.3).

\section{$4.3 \overline{w^{\prime 2}}$ Evolution Equation}

For stationary and two-dimensional flows, Eq. 5 applied to $\overline{w^{\prime 2}}$ reduces to

$$
\begin{aligned}
& 0=\underbrace{-\bar{U} \frac{\partial \overline{w^{\prime 2}}}{\partial x}}_{A D V} \underbrace{-\frac{2}{\rho_{0}} \overline{w^{\prime} \frac{\partial p^{\prime}}{\partial z}}}_{P C} \\
& \underbrace{-2 v \overline{\left(\left(\frac{\partial w^{\prime}}{\partial x}\right)^{2}+\left(\frac{\partial w^{\prime}}{\partial z}\right)^{2}\right)}}_{\varepsilon_{w^{\prime 2}}} \underbrace{-\frac{\partial \overline{u^{\prime} w^{\prime 2}}}{\partial x}-\frac{\partial \overline{w^{\prime 3}}}{\partial z}}_{T R}
\end{aligned}
$$

where the dynamical production term depends on $\bar{W}$ and can thus be neglected. Also, as with $\varepsilon_{u^{\prime 2}}$ for the $\overline{u^{\prime 2}}$ budget, $\varepsilon_{w^{\prime 2}}$ is estimated by Eq. 11 . The pressure-correlation term $(P C)$ is then again the only residual term of the measured budget of $\overline{w^{\prime 2}}$.

The $\overline{w^{\prime 2}}$ budget (Fig. 7e) shows that the turbulent transport term $(T R)$ is again weak, peaking near $z / \delta \cong 0.8$ as a source ( similar to $\overline{u^{\prime 2}}$ and $e$ ) but smaller in magnitude. The advection, also weak, peaks near $z / \delta \cong 0.8$ as a sink and similar to $\overline{u^{\prime 2}}$ and $e$. The main balance below $z / \delta \cong 0.8$ is between the pressure-correlation and the dissipation terms. In the outer layer, around $z / \delta=0.8$, the main source for $\overline{w^{\prime 2}}$ is thus the pressure-correlation term, as seen in the recent computations of Ashrafian and Andersson (2006). This observation confirms the previous hypothesis: the pressure fluctuations redistribute the energy from $\overline{u^{\prime 2}}$ to $\overline{w^{\prime 2}}$. It can also be observed that the $\overline{w^{\prime 2}}$ pressure-correlation term attains half the absolute value of the $\overline{u^{\prime 2}}$ pressure-correlation term, suggesting that the energy is equally partitioned between $\overline{w^{\prime 2}}$ and $\overline{v^{\prime 2}}$. This is confirmed by considering that the sum of the pressure-correlation terms of $\overline{u^{\prime 2}}$, $\overline{v^{\prime 2}}$ and $\overline{w^{\prime 2}}$ is zero (Eq. 8) since the pressure-correlation term of $e$ is assumed to be zero (see Sect. 4.1), which allows $P C \overline{v^{\prime 2}}$ to be evaluated. Yet, it is important to insist on the fact that the pressure-correlation terms for $\overline{u^{\prime 2}}$ and $\overline{w^{\prime 2}}$ are individually and independently estimated: each one is the residual of their respective budgets for the five downstream $x$ positions. Finally, 

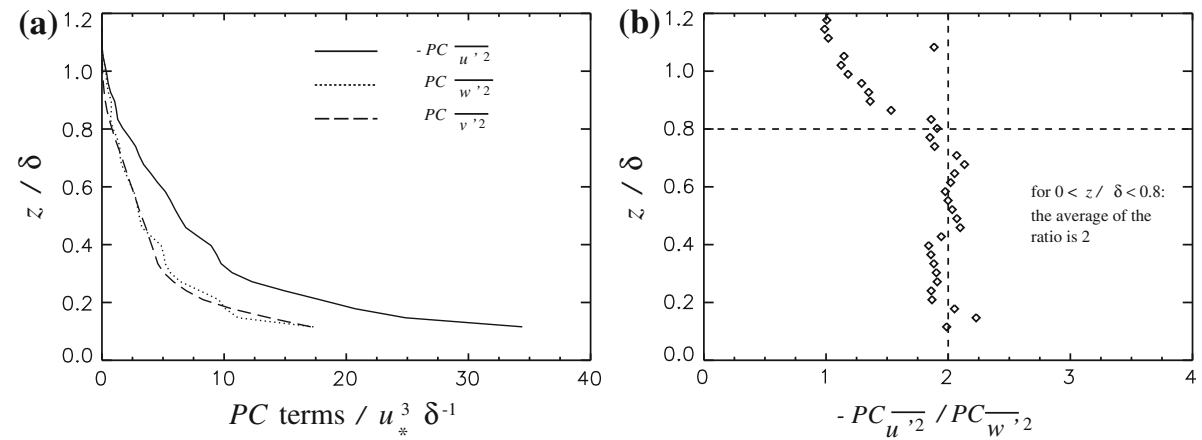

Fig. 8 (a) Locally normalised magnitude of the pressure-correlation terms $P C$ of the budget of $\overline{u^{\prime 2}}, \overline{w^{\prime 2}}$ and $\overline{v^{\prime 2}}$ as a function of $z / \delta$ for the D position $(x=6 \mathrm{~m})$. (b) Ratio of the normalised the pressure-correlation terms $-P C \overline{u^{\prime 2}} / P C \overline{w^{\prime 2}}$ as a function of $z / \delta$ for the $\mathrm{D}$ position $(x=6 \mathrm{~m})$

Fig. 7f shows the longitudinal evolution of the $P C \overline{w^{\prime 2}}$ term, and in agreement with all other terms it can be seen that equilibrium is reached for $x>3 \mathrm{~m}$.

Figure $8 \mathrm{a}$ shows all three pressure-correlation terms at $x=6 \mathrm{~m}$ while Fig. $8 \mathrm{~b}$ shows the ratio $-P C_{\overline{u^{\prime 2}}} / P C_{\overline{w^{\prime 2}}}$, and it can be seen in Fig. 8a that $P C_{\overline{v^{\prime 2}}}$ and $P C_{\overline{w^{\prime 2}}}$ are close for all $z / \delta$. The mean value of the ratio $-P C_{\overline{u^{2}}} / P C_{\overline{w^{\prime 2}}}$ in Fig. $8 \mathrm{~b}$ reveals a ratio close to 2 for $0<z / \delta<0.8$, and for $z / \delta>0.8$, the ratio surprisingly decreases close to 1 , but the magnitude of the terms is probably too small to obtain a significant estimate of the ratio. Awaiting more precise measurements for $z / \delta>0.8$, it can thus be concluded that the pressure fluctuations equitably distribute the energy contained in the longitudinal fluctuations to the transverse and vertical fluctuations:

$$
P C_{\overline{v^{\prime 2}}} \cong P C_{\overline{w^{\prime 2}}} \cong-\frac{1}{2} P C_{\overline{u^{\prime 2}}} .
$$

This redistribution, observed for all $x$ positions, is essentially the same as has been observed over a smooth-surface boundary layer (Pope 2000), suggesting that the turbulent structure in the outer layer is not influenced by the roughness elements.

\subsection{The $\overline{u^{\prime} w^{\prime}}$ Evolution Equation}

For $\overline{u^{\prime} w^{\prime}}$, under the stationarity and $2 \mathrm{D}$ assumptions, Eq. 5 reduces to

$$
\begin{aligned}
0= & \underbrace{-\bar{U} \frac{\partial \overline{u^{\prime} w^{\prime}}}{\partial x}}_{A D V}-\underbrace{-\left(\overline{u^{\prime 2}} \frac{\partial \bar{U}}{\partial x}+\overline{u^{\prime} w^{\prime}} \frac{\partial \bar{U}}{\partial z}\right)}_{D P} \\
& \underbrace{-\frac{1}{\rho_{0}}\left(\overline{u^{\prime} \frac{\partial p^{\prime}}{\partial z}}+\overline{w^{\prime} \frac{\partial p^{\prime}}{\partial x}}\right)}_{P C} \underbrace{-\frac{\partial \overline{u^{\prime 2} w^{\prime}}}{\partial x}-\frac{\partial \overline{u^{\prime} w^{\prime 2}}}{\partial z}}_{T R},
\end{aligned}
$$

where the dissipation term $\varepsilon_{u^{\prime}} w^{\prime}$ has been neglected (see Eq. 11), and the pressure-correlation term is thus the residual term here. The budget of $\overline{u^{\prime} w^{\prime}}$ is presented in Fig. $7 \mathrm{~g}$, which shows that the advection and turbulent transport terms are again relatively weak but with reversed signs compared to $\overline{u^{\prime 2}}, \overline{w^{\prime 2}}$ and $e$, and still peak near $z / \delta \cong 0.8$. Below, the main balance is between 
the dynamical production (source) and the pressure-correlation (sink), similar to the budget

of $\overline{u^{\prime 2}}$ where dissipation contributes as a sink. This result agrees with the measurements of Wyngaard (1992) and the simulations of Ashrafian and Andersson (2006). Wyngaard (1992) showed that the turbulent transport is negligible and that the pressure-correlation acts as a sink term that locally balances the dynamical production term. This repartition of energy was also observed by Mulhearn (1978) in wind-tunnel modelling of a rough-to-smooth boundary-layer transition. In conclusion, all budgets reach equilibrium between stations at $x=3 \mathrm{~m}$ and $x=6 \mathrm{~m}$ while the distribution of the budget terms is very similar to a smooth-surface turbulent boundary layer and to an internal boundary layer.

\section{Length Scales}

Here we investigate the dissipative length scale $l_{\varepsilon}$ and the mixing length $l_{m}$ based on the observed statistics, as well as the integral length scales based on spatial correlations. Both $l_{\varepsilon} \cong u^{3} / \varepsilon$ and $l_{m} \cong(K /(\partial \bar{U} / \partial z))^{1 / 2}$ are functional length scales, based on the ratio of statistical properties of the flow (Hunt et al. 1989), the former often being used to estimate the latter, which involves the sought-after Reynolds stress. Here, $u$ is a characteristic turbulent velocity, and $K$ is the turbulent eddy viscosity or exchange coefficient given by $K=-\overline{u^{\prime} w^{\prime}} /(\partial \bar{U} / \partial z)$ for horizontally homogeneous flow. First-order models are based on the so-called Prandtl-Kolomogorov relation, which assumes $K \cong u l$, where $u$ is most often taken as $e^{1 / 2}$ and $l$ is a length scale of the energy containing eddies, well described by the integral scale, but usually taken from a functional length scale. Our aim here is to characterise the turbulent scales of a neutral boundary-layer flow and to improve the parametrization of the functional length scales.

\subsection{Integral Length Scales}

Integral length scales can be obtained directly from spatial correlations with the present PIV measurements, without relying on the often necessary Taylor hypothesis. In particular, the spatial correlation function in the $(x, z)$ plane,

$$
R_{\overline{u_{i}^{\prime} u_{j}^{\prime}}}(\vec{r}, \overrightarrow{\delta r})=\frac{\overline{u_{i}^{\prime}(\vec{r}) u_{j}^{\prime}(\vec{r}+\overrightarrow{\delta r})}}{\overline{u_{i}^{\prime}(\vec{r}) u_{j}^{\prime}(\vec{r})}},
$$

can be estimated directly, where $\vec{r}=x \vec{i}+z \vec{k}$ is the reference position and $\vec{\delta} r$, taken as either $\delta x \vec{i}$ or $\delta z \vec{k}$, is the displacement, which is either positive or negative. The correlation functions are computed in each of the $N=999$ fluctuating $(x, z)$ velocity fields with $\vec{r}$ for all measured points in the velocity fields. The resulting correlation functions are then averaged over all $N$ for convergence.

The integral length scales at each reference $x$ position are then computed via the integral of the correlation functions (Eq. 15):

$$
L_{\overline{u_{i}^{\prime} u_{j}^{\prime}}, \delta \vec{r}}(\vec{r})=\int_{0}^{\infty} R_{\overline{u_{i}^{\prime} u_{j}^{\prime}}}(\vec{r}, \overrightarrow{\delta r}) d \overrightarrow{\delta r} .
$$

To ensure systematic convergence, the integration for large $r$ was terminated when the correlation function reached 0.1 . The measurements permit 16 different integral scales to be obtained, i.e. for the four stresses $\left(\overline{u^{\prime 2}}, \overline{w^{\prime 2}}, \overline{u^{\prime} w^{\prime}}\right.$ and $\overline{w^{\prime} u^{\prime}}$ ), each in two directions $(\delta x \vec{i}$ and 

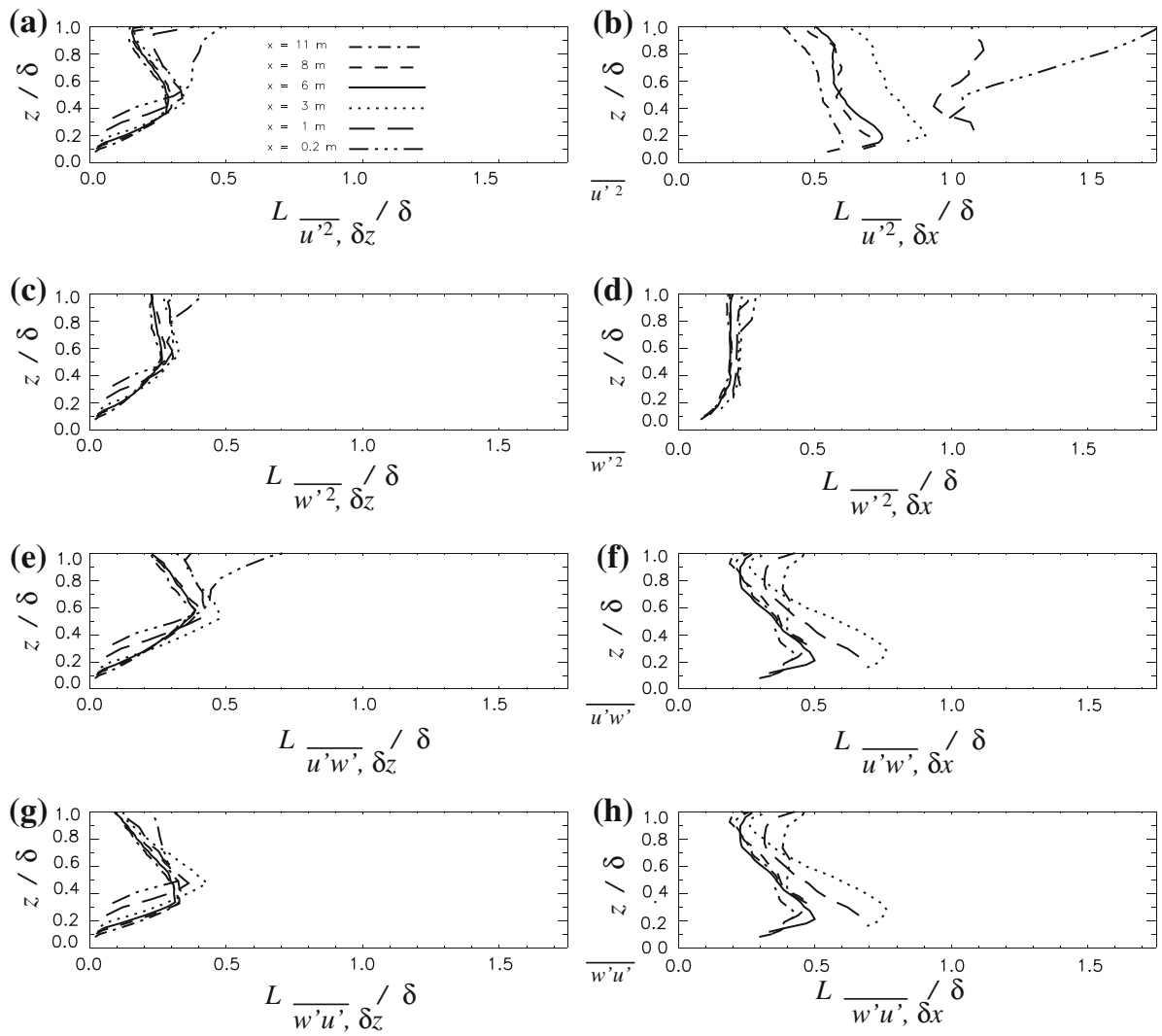

Fig. 9 Normalised minimum vertical and longitudinal integral length scale $L \overline{u_{i}^{\prime} u_{j}^{\prime}}, \delta z$, in the left column

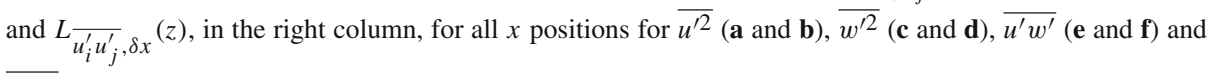
$\overline{w^{\prime} u^{\prime}}(\mathbf{g}$ and $\mathbf{h})$

$\delta z \vec{k})$, with $\delta x$ and $\delta z$ either positive or negative. As expected, it was observed that $\delta x$ either positive or negative does not affect $L_{\overline{u_{i}^{\prime} u_{j}^{\prime}}, \delta x}(\vec{r})$, emphasising that the flow is homogenous in $x$, at least at the scale of the measurement fields. However, for $L_{\overline{u_{i}^{\prime} u_{j}^{\prime}}, \delta z}(\vec{r})$, the results revealed a general tendency for larger integral scales in the top half of the boundary layer for negative $\delta z$ displacements than for positive ones, and vice versa for the bottom half. Since Carlotti and Drobinski (2004) argued that the smallest vertical integral scales should be close to the mixing length, the minimum integral-scale value between the positive and negative $\delta z$ displacements at each $z$ level (for all four stresses) was chosen. The resulting integral scales are regrouped in Fig. 9a-h for all $x$ positions, and it can be seen that equilibrium of the integral scales is also reached between $3 m<x<6 \mathrm{~m}$, as for the turbulent statistics and budgets. Nevertheless, the first $x$ positions $(x=0.2 \mathrm{~m}$ and $x=1 \mathrm{~m})$ exhibit the same tendencies except for $L_{\overline{u^{\prime 2}}, \delta x}$. It can also be noted that $L_{\overline{u^{\prime 2}}, \delta x}$ and $L \overline{u^{\prime} w^{\prime}}, \delta x$ reveal a greater gap between $x=3 \mathrm{~m}$ and $x=6 \mathrm{~m}$, illustrating that $u^{\prime}$ is more affected by the growth of the boundary layer than $w^{\prime}$, which is more associated with the local turbulence. Also, it can be observed that $L \overline{u^{\prime 2}}, \delta x$ dominates all other length scales, as expected; this dominance of $L \overline{u^{\prime 2}, \delta x}$ 

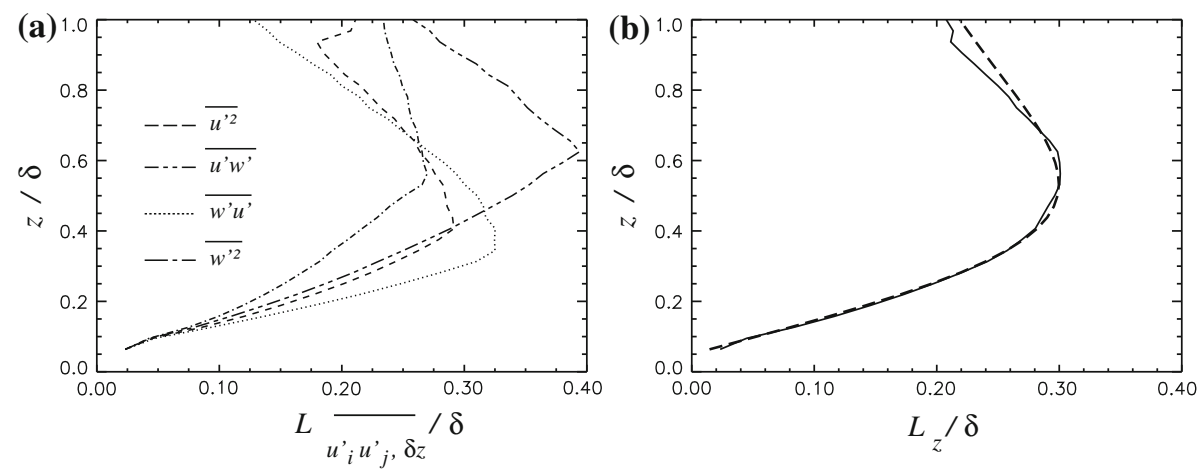

Fig. 10 (a) Normalised minimum vertical integral length scales for $\overline{u^{\prime 2}}, \overline{w^{\prime 2}}, \overline{u^{\prime} w^{\prime}}$ and $\overline{w^{\prime} u^{\prime}}$. (b) Mean vertical integral length scale $\left(L_{z}\right)$ of $(\mathbf{a})$ and its fit with $L_{z} / \delta=\left[A\left(z-z_{h} / \delta-s\right)\right] /\left[B+\left(\frac{z-z_{h}}{\delta}-s\right)^{p}\right]$, with $A=0.26$, $s=0.05, p=2.4$ and $B=0.24$

is due to the existence of coherent structures that are elongated in the longitudinal direction (e.g. Drobinski et al. 2007). These structures are localised in the bottom part of the boundary layer with a maximum at $z / \delta=0.2$, which can also be observed in the $L \overline{u^{\prime} w^{\prime}}, \delta x(z)$ profiles.

For 1D modelling purposes, it is more appropriate to consider the vertical scales, given by $\delta z$ displacements. As seen in the left column of Fig. 9, all the vertical integral scales increase

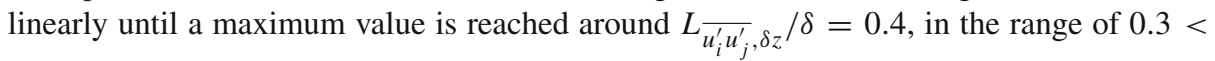
$z / \delta<0.6$. Above, the vertical integral scales decrease to $L \overline{w^{\prime 2}}, \delta z=0.2, L \overline{u^{\prime} w^{\prime}}, \delta z / \delta=0.2$ and $L \overline{w^{\prime} u^{\prime}, \delta z} / \delta=0.1$ at $z / \delta=1$. Only $L \overline{u^{\prime 2}}, \delta z$ increases again above $z / \delta=0.9$, which underlines the fact that significant exchanges of longitudinal structures occur between the boundary layer and the free stream. The vertical velocity fluctuation scales $L \overline{w^{12}, \delta z}$ and $L \overline{w^{12}}, \delta x$ are very close, however, suggesting that the length scales of $\overline{w^{\prime 2}}$ are essentially isotropic.

Figure 10a compares directly the profiles of the resulting vertical integral scales of the four stresses. Here, it can be seen more clearly that the vertical integral length scales essentially have the same shape and intensity, increasing in the bottom half of the boundary layer and decreasing above. More precisely, below $z / \delta \leq 0.2$ in the inertial sublayer, all profiles collapse very well, but above there are differences. Also, as expected due to the inhomogeneity in the $z$-direction, the integral scales of the $\overline{u^{\prime} w^{\prime}}$ and $\overline{w^{\prime} u^{\prime}}$ fluxes are unequal, with a marked shift in the maximum scale.

To compare the integral length scales with $l_{m}$ and $l_{\varepsilon}$ used in $1 \mathrm{D}$ prediction models at equilibrium, a representative integral length scale $\left(L_{z}\right)$ was taken as the average of the four vertical length scales in Fig. 10a. This mean integral length scale $\left(L_{z}\right)$, shown in Fig. 10b, is linear in the lower part of the outer layer, as expected. Above, $L_{z}$ tends to be hyperbolic, and the maximum value of the integral scale is $L_{z} / \delta \cong 0.3$ at $z / \delta=0.5$. A function of the form $a z /\left(b+z^{k}\right)$ is thus expected to be adapted, as the dashed line in Fig. 10b shows. The fitted normalised relation is:

$$
\frac{L_{z}}{\delta}=\frac{A\left(\frac{z-z_{h}}{\delta}-s\right)}{B+\left(\frac{z-z_{h}}{\delta}-s\right)^{p}},
$$

with $A=0.26, s=0.05, p=2.4$ and $B=0.24$. 

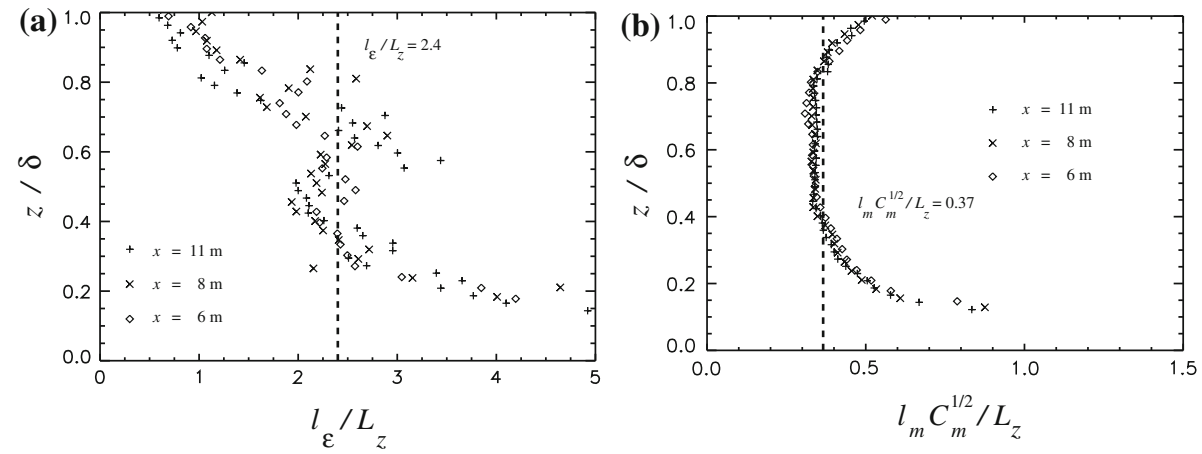

Fig. 11 (a) Ratio of the integral length scale and the dissipative length scale $\left(l_{\varepsilon} / L_{z}\right)$ at the developed $x$ positions as a function of $z / \delta$. (b) Ratio of the integral lengthscale and the mixing length $\left(l_{m} C_{m}^{1 / 2} / L_{z}\right)$ at developed $x$ positions as a function of $z / \delta$

\subsection{Dissipative Length Scales}

In the following sections, we consider only the region where the flow has reached equilibrium, i.e. for $x>6 \mathrm{~m}$. Taylor (1935) first suggested that $\tilde{\varepsilon} \cong u^{3} / l$, usually written as:

$$
\tilde{\varepsilon}=C_{\varepsilon} \frac{e^{3 / 2}}{l_{\varepsilon}},
$$

where $C_{\varepsilon}$ is a constant $\approx 0.85$ (Schmidt and Schumman 1989; Cuijpers and Duynkerke 1993; Canuto et al. 2001; Cheng et al. 2002). Using the dissipation rate obtained from the budget of $e$ (Eq. 9), $l_{\varepsilon}$ can thus be determined via Eq. 18. Figure 11a shows the resulting vertical profile of $l_{\varepsilon}$ normalised by the experimental integral length $L_{z}$ of Fig. 10b. Clearly, except between $0.3<z / \delta<0.7$, where $l_{\varepsilon} \cong 2.4 L_{z}, l_{\varepsilon} / L_{z}$ is not constant, which is not surprising since the two scales should only be proportional in homogeneous turbulence.

\subsection{Mixing Length}

The mixing length model,

$$
\overline{u^{\prime} w^{\prime}}=-C_{m} l_{m}^{2}\left|\frac{\partial \bar{U}}{\partial z}\right| \frac{\partial \bar{U}}{\partial z},
$$

where $C_{m}$ is a constant, is used to model the Reynolds stress when one length scale dominates. It can be used here to deduce the mixing length $l_{m}$ from the measured mean velocity gradient and Reynolds stress profile, also for $x>6 \mathrm{~m}$. Figure $11 \mathrm{~b}$ shows the resulting normalised profile $l_{m} C_{m}^{1 / 2} / L_{z}$. In comparison with the dissipative length scale ratio, $l_{\epsilon} / L_{z}$, the mixing length $l_{m}$ matches $L_{z}$ better. Neglecting the strong nonlinearities near the surface for $z \leq 0.1 \delta$, the scales are approximately proportional over a wider range, between $0.3<z / \delta<0.9$, with a ratio of proportionality $\left(l_{m} C_{m}^{1 / 2} / L_{z}\right)$ of about 0.37 . The standard mixing length in the logarithmic layer,

$$
l_{m}=\kappa\left(z-z_{d}\right),
$$

in combination with $l_{m} C_{m}^{1 / 2}$ determined from Eq. 19, permits $C_{m}$ to be determined in the log-law region via linear regression, yielding an average $C_{m}=0.51$ for the three latest $x$ 


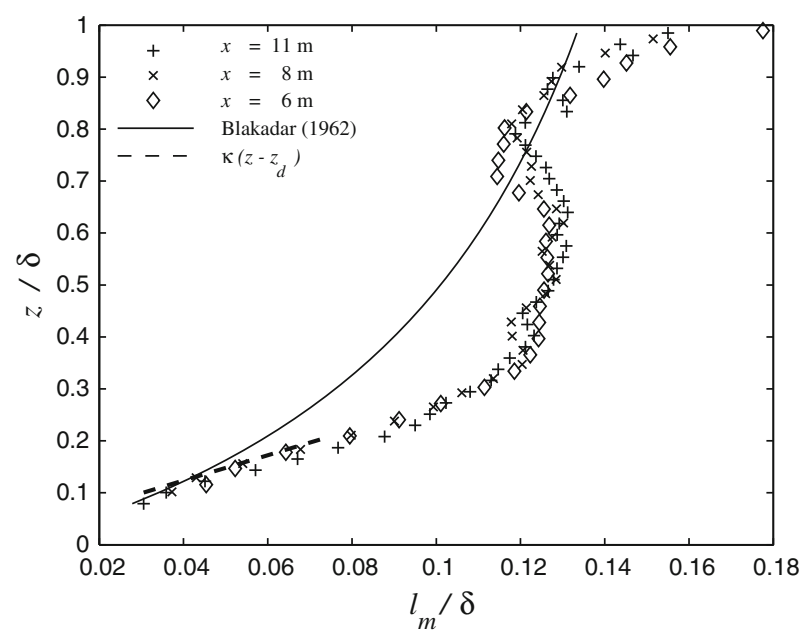

Fig. 12 Measured mixing length $l_{m} / \delta$ deduced from Eq. 19 (symbols) at developed $x$ positions as a function of $z / \delta$, compared to Eq. 20, dashed line, $l_{m}=\kappa(z-d)$, and Eq. 21, solid line $l_{m}=l_{0} \frac{\kappa z}{\kappa z+l_{0}}$ (Blackadar 1962)

stations. Thus, the ratio of proportionality $l_{m} / L_{z}$ for $0.3 \leq z / \delta \leq 0.9$ is about 0.52 . Figure 12 shows $l_{m} / \delta$ with $C_{m}=0.51$ as well as the standard mixing length in the logarithmic layer (Eq. 20). The formulation of Blackadar (1962),

$$
l_{m}=l_{0} \frac{\kappa z}{\kappa z+l_{0}},
$$

is also shown in Fig. 12, by determining $l_{0}$ from a least squares fit of $l_{m}$ for the last three $x$ stations. The resulting three $l_{0} / \delta$ ratios are very close, with an average of 0.24 . It can be seen that the normalized measurements for all three stations follow essentially the same curve, which might be described as a weak $S$ shape. In the central outer layer, between $0.3 \leq z / \delta \leq 0.7$, $l_{m} / \delta$ is largely constant at about 0.13 , before retreating slightly and then increasing as the top of the boundary layer is approached. Not surprisingly, Blackadar's relation used for all stability conditions does not fit the data well. A parametrization based on either the dissipative scale or the vertical integral scale would also yield significant differences, so direct parametrization of the mixing length based on the measured $l_{m}$ profiles in Fig. 12 might be most appropriate.

\section{Conclusion}

The main goal of our study was to investigate turbulence in the neutral boundary layer to help validate numerical simulation models and to help improve the parametrizations of the turbulent processes for one-dimensional neutral boundary-layer models. The experiment presented herein reproduces the development of a neutral boundary layer in a water flume over a rough surface at high Reynolds numbers. The velocity measurements were performed via a two-dimensional particle image velocimetry (PIV) technique in the vertical symmetry plane of the flow. The turbulent statistics at several longitudinal positions were obtained by spatially and temporally averaging the PIV velocity fields, ensuring statistical convergence for the higher-order statistics. 
The boundary-layer growth was established on the basis of the mean longitudinal velocities and the turbulent shear stress, yielding a 0.8 power law in agreement with previous results for the growth of an internal boundary layer. However, it appears that our 0.18 coefficient is smaller than that for a typical internal boundary layer. The commencement of the roughness surface is well revealed by mean vertical velocity perturbations whose streamwise extent is about $0.18 \mathrm{~m}$.

An analysis of the budget of turbulent kinetic energy $(e)$ shows that shear production is the main source of turbulence in the outer layer, as is the case for the turbulent boundary layer over a smooth surface. The residual term in this budget yielded the dissipation rate, allowing also the dissipation of the $\overline{u^{\prime 2}}$ and $\overline{w^{\prime 2}}$ budgets to be estimated. Finally, we were able to quantify the pressure fluctuations and describe their role in transferring energy between the different variances. Again, these transfers were found to mirror the exchanges due to the pressure-correlation terms in a smooth boundary layer, suggesting the turbulent structure of the outer layer over a rough surface is very similar to that over a smooth surface. The development of the rough-surface neutral boundary layer does not significantly modify the repartition between the constituent terms in the budget of $e, \overline{u^{\prime 2}}, \overline{w^{\prime 2}}$ and $\overline{u^{\prime} w^{\prime}}$. Whatever the variable, even before equilibrium is reached, the same terms govern the transfer of energy, except for the vertical turbulent transport of $e$, which is a minor term when $0.1<z / \delta<0.6$ at all stations except for $x=1 \mathrm{~m}$, where it is not negligible, and where it appears as a sink of energy that balances the "surplus" of dynamical production. All budgets, as well the first-order and second-order statistics reach equilibrium between $12,000 \leq x / z_{0} \leq 24,000$.

Finally, the spatially resolved measurements allowed us to compute the spatial integral length scales directly, including the vertical scales, that are more appropriate for modelling purposes. These integral length scales were used to compare against the dissipative and the mixing length functional scales. It is shown that the ratio of the dissipative scale to the vertical integral length scale, not expected to be constant in this inhomogeneous flow, indeed varies significantly by a factor of up to 2 . Direct calculation of the mixing length suggests a better match with the vertical integral scale, with a narrow constant of proportionality for $z / \delta \geq 0.1$ of about $l_{m} / L_{z} \cong 0.52$. Further numerical tests need to be done in order to validate the new proposal for $L_{z}$ and to verify whether this improves the simulation of the neutral boundary layer.

Acknowledgments This work study was supported by the MesoScale Meteorology and Experiment Meteorology groups (GMME and GMEI) of the National Center of Meteorological Research of Météo-France. The authors would like to thank B. Beaudoin, J-C. Boulay, J.-C. Canonici, M. Morera, S. Lassus Pigat and H. Schaffner of the CNRM-GAME fluid mechanics laboratory (SPEA) for their support in the experiments and for providing us with valuable comments.

Open Access This article is distributed under the terms of the Creative Commons Attribution Noncommercial License which permits any noncommercial use, distribution, and reproduction in any medium, provided the original author(s) and source are credited.

\section{References}

Antonia RA, Luxton RE (1971) The response of a turbulent boundary layer to a step change in surface roughness. Part 1. smooth to rough. J Fluid Mech 48:721-761

Ashrafian A, Andersson HI (2006) Roughness effects in turbulent channel flow. Int J Prog Comput Fluid Dyn 6:1-20

Bandyopadhyay PR (1987) Rough-wall turbulent boundary layers in the transition regime. J Fluid Mech 180:231-266 
Belcher SE, Jerram N, Hunt JCR (2003) Adjustment of a turbulent boundary layer to a canopy of roughness elements. J Fluid Mech 488:369-398

Blackadar AK (1962) The vertical distribution of wind and turbulent exchange in a neutral atmosphere. J Geophys Res 67(8):3095-3102

Blackadar AK (1965) A single layer theory of the vertical distribution of wind in a baroclinic neutral atmospheric boundary layer. Final report, AFCRL-65-531, Department of Meteorology, The Pennsylvania State University, University Park, PA, 22 pp

Bottema M (1995) Parameterisation of aerodynamic roughness parameters in relation to air pollutant removal efficiency of streets. In: Proceedings of 3rd international conference on Air pollution, Wessex Institute of Techn, Porto Carras, Greece, pp 235-242

Bottema M (1997) Urban roughness modelling in relation to pollutant dispersion. Atmos Environ 31:30593075

Bougeault P, Lacarrère P (1989) Parameterization of orography-induced turbulence in a meso-beta scale model. Mon Weather Rev 117:1870-1888

Bouttier F (2003) The AROME mesoscale project. In: Seminar on Recent developments in data assimilation for atmosphere and ocean, 8-12 September, ECMWF, European Center for Medium-Range Weather Forecasts

Bouttier F (2007) Arome, avenir de la prévision régionale. La Météorol 58:12-20

Burattini P, Leonardi S, Orlandi P, Antonia RA (2008) Comparison between experiments and direct numerical simulations in a channel flow with roughness on one wall. J Fluid Mech 600:403-426

Canuto VM, Cheng Y, Howard A (2001) New third-order moments for the convective boundary layer. J Atmos Sci 58:1169-1172

Carlotti P, Drobinski P (2004) Length scales in wall-bounded high-Reynolds-number turbulence. J Fluid Mech 516:239-264

Castro IP (2007) Rough-wall boundar layers: mean flow universality. J Fluid Mech 585:469-485

Castro IP, Cheng H, Reynolds R (2006) Turbulence over urban-type roughness: deductions from wind-tunnel measurements. Boundary-Layer Meteorol 118:109-131

Cheng H, Castro IP (2002a) Near-wall flow development after a step change in surface roughness. BoundaryLayer Meteorol 105:411-432

Cheng H, Castro IP (2002b) Near-wall flow over urban-like roughness. Boundary-Layer Meteorol 104: 229-259

Cheng Y, Canuto VM, Howard AM (2002) An improved model for the turbulent PBL. J Atmos Sci 59: 1550-1565

Chow FK, Street RL, Xue M, Ferziger JH (2005) Explicit filtering and reconstruction turbulence modeling for large-eddy simulation of neutral boundary layer flow. J Atmos Sci 62:2058-2077

Coceal O, Belcher SE (2004) A canopy model of mean winds through urban areas. Q J Roy Meteorol Soc 130:1349-1372

Coceal O, Thomas TG, Castro IP, Belcher SE (2006) Mean flow and turbulence statistics over groups of urban-like cubical obstacle. Boundary-Layer Meteorol 121(3):475-490

Cuijpers JWM, Duynkerke PG (1993) Large eddy simulation of trade wind cumulus clouds. J Atmos Sci 50:3894-3908

Drobinski P, Carlotti P, Newson RK, Banta RM, Foster RC, Redelsperger JL (2004) The structure of nearneutral surface layer. J Atmos Sci 61:699-714

Drobinski P, Carlotti P, Redelsperger JL, Banta RM, Masson V, Newson RK (2007) Numerical and experimental investigation of the neutral atmospheric surface layer. J Atmos Sci 64:137-156

Ducrocq V, Bouttier F, Malardel S, Montmerle T, Seity Y (2005) Le projet arome. La Houille Blanche 2:39-43

Elliott W (1958) The growth of the atmospheric internal boundary layer. Trans Am Geophys Union 39:10481054

Fincham AM, Delerce G (2000) Advanced optimization of correlation imaging velocimetry algorithms. Exp Fluids 39(suppl):S13-S22

Fincham AM, Spedding GR (1997) Low cost, high resolution DPIV for measurement of turbulent fluid flow. Exp Fluids 23:449-462

Flack KA, Schultz MP, Shapiro TA (2005) Experimental support for Towsend's Reynolds number similarity hypothesis on rough walls. Phys Fluids 17(3):1-9

Freeman FR, Jacobson MZ (2002) Transport-dissipation analytical solutions of the $e-\epsilon$ turbulence model and their role in predictions of the neutral ABL. Boundary-Layer Meteorol 102:117-138

Glendening JW, Lin CL (2002) Large eddy simulation of internal boundary layers created by a change in surface roughness. J Atmos Sci 59:1697-1711

Grant ALM (1986) Observations of boundary layer structure made during the 1981 Kontur experiment. Q J Roy Meteorol Soc 112:825-841 
Hess GD, Garratt JR (2002) Evaluating models of the neutral, barotropic planetary boundary layer using integral measures. Part II. modelling observed conditions. Boundary-Layer Meteorol 104:359-369

Hommema SE, Adrian RJ (2003) Packet structure of surface eddies in the atmospheric boundary layer. Boundary-Layer Meteorol 106:35-60

Hunt JCR, Moin P, Lee M, Moser RD, Sparlat P, Mansour NN, Kaimal JC, Gaynor E (1989) Cross-correlation and length-scales in turbulent flows near surfaces. In: Fernholz HH, Fiedler HE (eds) Advances in turbulences, vol 2. Springer, Berlin, pp 128-134

Jiménez J (2004) Turbulent flows over rough walls. Annu Rev Fluid Mech 36:173-196

Kim J, Moin P, Moser R (1987) Turbulence statistics in fully developed channel flow at low Reynolds number. J Fluid Mech 177:133-166

Kondo J, Yamazawa H (1986) Aerodynamic roughness over an inhomogeneous ground surface. BoundaryLayer Meteorol 35:331-348

Krogstad PA, Antonia RA, Browne LWB (1992) Comparison between rough- and smooth-wall turbulent boundary layers. J Fluid Mech 245:599-617

Krogstad PA, Andersson HI, Bakken OM, Ashrafian A (2005) An experimental and numerical study of channel flow with rough walls. J Fluid Mech 530:327-352

Kunkel GJ, Marusic I (2006) Study of the near wall turbulent region of the high Reynolds number boundary layer using an atmospheric flow. J Fluid Mech 548:375-402

Lafore JP, Stein J, Asencio N, Bougeault P, Ducrocq V, Duron J, Fisher C, Hereil P, Mascart P, Masson V, Pinty JP, Redelsperger JL, Richard E, Gueraude Arellano JV (1998) The Meso-NH atmospheric simulation system. Part I: adiabatic formulation and control simulations. Ann Geophys 16:90-109

Lettau HH (1962) Theoretical wind spirals in the boundary layer of a barotropic atmosphere. Beitr Phys Atmos $35: 195-212$

Lettau H (1969) Note on aerodynamic roughness parameter estimation on the basis of roughness element description. J Appl Meteorol 8:828-832

Lin CL, Moeng CH, Sullivan PP, McWilliams JC (1997) The effect of surface roughness on flow structures in a neutrally stratified planetary boundary layer flow. Phys Fluids 11:3235-3249

Macdonald RW (2000) Modelling the mean velocity profile in the urban canopy layer. Boundary-Layer Meteorol 97:25-45

Macdonald RW, Griffiths RF, Hall DJ (1998) An improved method for estimation of surface roughness of obstacle arrays. Atmos Environ 32:1857-1864

Macdonald RW, Schofield SC, Slawson PR (2002) Physical modelling of urban roughness using arrays of regular roughness elements. Water Air Soil Pollut Focus 2:541-554

Marusic I, Kunkel GJ (2003) Streamwise turbulence intensity formulation for flat-plate boundary layers. Phys Fluids 15:2461-2464

Mason PJ, Thomson DJ (1987) Large-eddy simulation of the neutral static stability planetary boundary layer. Q J Roy Meteorol Soc 113:413-442

Moeng CH, Sullivan PP (1994) A comparison of shear- and buoyancy-driven planetary boundary layer flows. J Atmos Sci 51:999-1022

Mulhearn PJ (1978) A wind-tunnel boundary layer study of the effects of a surface roughness change: rough to smooth. Boundary-Layer Meteorol 15:3-30

Nicholls S, Readings CJ (1979) Aircraft observations of the structure of the lower boundary layer over the sea. Q J Roy Meteorol Soc 105:785-802

Panofsky HA (1974) The atmospheric boundary layer below 150 meters. Annu Rev Fluid Mech 6:147-177

Pendergrass W, Arya PS (1984) Dispersion in neutral boundary layer over a step change in surface roughness-I. Mean flow and turbulence structure. Atmos Environ 18(7):1267-1279

Perrier M, Butet A (1988) Veine hydraulique du cnrm: fonctionnement en écoulement neutre. Note de travail de l'etablissement d'etudes et de recherches météorologique n 216, Ministère des Transports et de la Mer, Direction de la Météorologie Nationale, 54 pp

Pope SB (2000) Turbulent flows. Cambridge University Press, Cambridge, 771 pp

Poulos GS et al (2002) Cases-99: a comprehensive investigation of the stable nocturnal boundary layer. Bull Am Meteorol Soc 83:555-581

Rao K, Wyngaard J, Coté O (1974) The structure of the two dimensional internal boundary layer over a sudden change of surface roughness. J Atmos Sci 31:738-746

Raupach MR, Thom AS, Edwards I (1980) A wind-tunnel study of turbulent flow close to regularly arrayed rough surfaces. Boundary-Layer Meteorol 18:373-397

Raupach MR, Antonia RA, Rajagopalan S (1991) Rough-wall boundary layers. Appl Mech Rev 44:1-25

Redelsperger JL, Mahé F, Carlotti P (2001) A simple general subgrid model suitable both for surface layer and free-stream turbulence. Boundary-Layer Meteorol 101:375-408 
Saddoughi SG (1997) Local isotropy in complex turbulent boundary layer at high Reynolds number. J Fluid Mech 348:201-245

Saddoughi SG, Veeravalli SV (1994) Local isotropy in turbulent boundary layer at high Reynolds number. J Fluid Mech 268:333-372

Schlichting U, Gersten K (1979) Boundary layer theory, 8th revised and enlarged edition 2000. Springer, Berlin, $817 \mathrm{pp}$

Schmidt H, Schumman U (1989) Coherent structure of the convective boundary layer derived from large eddy simulations. J Fluid Mech 200:511-562

Schultz MP, Flack KA (2007) The rough-wall turbulent boundary layer from the hydraulically smooth to the fully rough regime. J Fluid Mech 580:381-405

Seity Y, Brousseau P, Malardel S, Hello G, Bénard P, Bouttier F, Lac C, Masson V (2010) The AROME-France convective scale operational model. Mon Weather Rev. doi:10.1175/2010MWR3425.1

Spalart PR (1988) Direct simulation of a turbulent boundary layer up to $r_{\theta}=1410$. J Fluid Mech 187:61-98

Taylor GI (1935) Statistical theory of turbulence: parts I-III. Proc Roy Soc Lond A 151:421-464

Tennekes H, Lumley JL (1972) A first course in turbulence. MIT Press, Cambridge, MA, 300 pp

Townsend AA (1966) The flow in a boundary layer after a change in surface roughness. J Fluid Mech 26: 255-266

Townsend AA (1976) The structure of turbulent shear flow. Cambridge University Press, 429 pp

White FM (1991) Viscous fluid flow, 2nd edn. McGraw-Hill International Editions, New York, 614 pp

Wyngaard JC (1992) Atmospheric turbulence. Annu Rev Fluid Mech 24:205-233

Xu D, Taylor PA (1997a) An $e-\epsilon$ turbulence closure scheme for planetary boundary-layer models: the neutrally stratified case. Boundary-Layer Meteorol 84:247-266

Xu D, Taylor PA (1997b) On turbulence closure constants for atmospheric boundary-layer modelling: neutral stratification. Boundary-Layer Meteorol 84:267-287

Yaglom AM (1991) Similarity laws for wall turbulence flows. In: Dracos T, Tsinober A (eds) New approaches and concept in turbulence. Monte Veritá, Birkhauser, 20 pp 